\title{
A Comparative Study on Performance and Reliability of 32-bit Binary Adders
}

\author{
Milad Bahadori ${ }^{1}$, Mehdi Kamal ${ }^{1}$, Ali Afzali-Kusha ${ }^{1}$, Massoud Pedram ${ }^{2}$ \\ ${ }^{1}$ School of Electrical and Computer Engineering, University of Tehran, Tehran, Iran \\ ${ }^{2}$ Department of Electrical Engineering, University of Southern California, Los Angeles, CA, USA \\ \{milad.bahadori, mehdikamal, afzali\}@ut.ac.ir, pedram@usc.edu
}

\begin{abstract}
In this paper, the performance and reliability of different binary adder families are studied for both the superthreshold and the near-threshold regions of operation. The adder structures are selected from both the carry propagate adders (CPAs) and parallel prefix adders (PPAs). The performance parameters which are used in the comparative study include delay, power, energy, and energy-delay-product (EDP) of the adders. Additionally, the impacts of the process variation and negative bias temperature instability (NBTI) on the delays of the adders under the aggressive supply voltage scaling are investigated. Also, the efficacies of the adders are compared using a merit function based on their performance and reliability parameters for a wide range of supply voltage levels, from the nominal voltage down to the near-threshold voltage. The study is performed for the 32-bit adder structures designed based on the 14-nm FinFET and 45-nm bulk CMOS technologies. The results which are obtained using HSPICE simulations, reveal that the reliability parameters similar to the performance parameters are a function of the adder architectures and those are the key components to determine the efficiencies of the adders. Also, the results show that the impacts of the process variation and NBTI on the delays of the high performance PPA structures are more than those of the CPA structures for the whole range of the supply voltage. The PPAs, however, have the higher merit factors compared to the CPAs under a wide range of supply voltage levels. The results presented in this paper may provide some guidelines for the designers to select proper adder structures based on their design requirements and constraints.
\end{abstract}

Keywords - Binary adders, aggressive supply voltage scaling, superthreshold, near-threshold, performance, reliability, process variation, negative bias temperature instability (NBTI).

\section{INTRODUCTION}

Adders, as fundamental blocks in arithmetic and logic units (ALUs), perform different operations such as subtraction, multiplication, division, and, address computation as well as additions [1][2]. These units are one of the most power hungry components in the processors and often are the possible location of hot-spots [3]. On the other hand, obtaining high operation speeds at low power consumptions which is a desirable objective makes the design of these blocks very challenging. In fact, the power consumption has become the primary design constraint for chip designers [4]. Scaling the feature sizes in the circuit through fabrication advancement has been one of the key knobs for improving the speed and power consumption. However, due to the fabrication process limitations in advanced technologies, the physical parameters of the circuit components such as those of the devices and interconnects mostly become different than those which were initially intended [5]. Random dopant fluctuation (RDF) and line edge roughness (LER) are two main (dominant) sources of the process variation [6]. These variations cause variations in the merit (e.g., speed and power consumption) parameters of the same circuit located in different parts of one single wafer or fabricated on the same part of the wafer at different times. These cause zero time variations of one circuit parameters compared to those of the same circuit on a different die reducing the fabrication yield of a circuit. In addition to this, when the circuit age increases, the circuit parameters become different than those of the time zero ones due to phenomena such as bias temperature instability (BTI) [5]. While the former which is sort of spatial variation affects the yield of the fabrication process, the latter is a temporal variation determining the lifetime of the circuit. The impacts of the process variation and BTI on the circuit fabrication yield and lifetime are affected by the circuit structure. Hence, in a comparative study of the digital circuits with the same function, in addition to the delay (speed) and power/energy consumption, these impacts should be included in the comparative study.

One of the effective techniques to lower the power consumption of digital circuits is to reduce the supply voltage which may take the operation of the $\mathrm{ON}$ device from the superthreshold to sub-threshold regions with the nearthreshold one in the middle [7]. Due to quadratic voltage dependence of the switching energy and the exponential voltage dependence of the subthreshold current, this reduction gives rise to the both dynamic and static power reductions [3]. The subthreshold current is also the charging current when the device operates in the sub-threshold region of operation and is the main leakage component when the device is OFF in all regions of operation. The exponential dependence originates mainly from the drain induced barrier lowering (DIBL) effect [7]. Therefore, reducing the circuit supply voltage reduces both the dynamic and static (leakage) powers of the digital circuits. While the reduction lowers the impact of the BTI, it is achieved at the price of increased delay and sensitivity to the process variation [4]. Working in the superthreshold region provides us with lower delay and higher switching and leakage powers compared to the near/sub-threshold regions. In the sub-threshold region, the logic gate delay and leakage power exhibit exponential dependences on the supply and threshold voltages (Fig. 1). The near-threshold region provides a lower delay and less sensitivity to the process and environmental variations compared to that of the sub-threshold region and significantly lower switching and leakage powers compared to those of the superthreshold region (Fig. 1) [4]. In addition, the design of the circuits with the feature of dynamic voltage and frequency scaling (DFVS), for the applications where the 


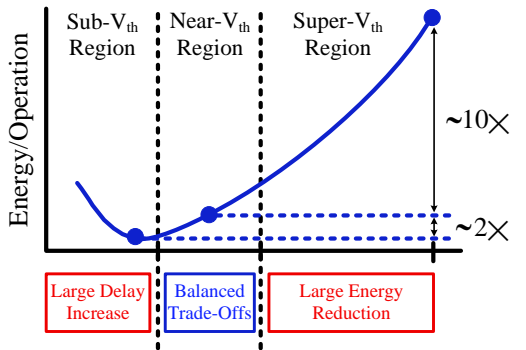

(a)

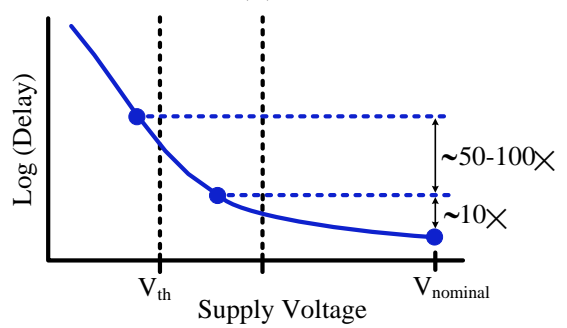

(b)

Fig. 1. (a) Energy and (b) delay trends versus the supply voltage in different regions of operation [4].

circuit speed requirement is dynamic, may allow for a considerable power and energy reduction [8]. Therefore, for a better comparison of the circuits, the impact of the supply voltage scaling also should be included in the investigation.

In this paper, we perform a comparative study on speed, power/energy consumption, area, impact of the process variation, and negative BTI (NBTI) induced delay degradation of some of the conventional 32-bit binary adders. The study is performed for a wide range of supply voltages to investigate the performances of the adders when they operate in both the superthreshold and near-threshold regions. The investigation is performed for both 14-nm FinFET and 45-nm bulk CMOS technologies. The rest of the paper is organized as follows. In Section II, some of the works related to the comparative study of the adders which have focused on the performance and reliability parameters are discussed. The structures of the adders considered in this work are briefly described in Section III. The impact of the supply voltage scaling on the delays and powers of the adders are investigated in Section IV. In addition, the effect of the scaling on the delays of the adders in the presence of the process variation and NBTI are studied in Section V. Section VI presents a discussion on comparing the adders based on their performance and reliability parameters in the presence of the process variation and NBTI aging effects under a wide range of supply voltage levels. Finally, the paper is concluded in Section VII.

\section{RELATED WORKS}

Many adder families/structures with different speeds, power consumptions, and area usages have been introduced in the literature (e.g., [9]-[14]). Among them ripple carry adder (RCA) has the simplest structure compared to those of the other adders leading to low power consumption and area usage at the price of high delay [2]. Different structures include carry skip adder (CSKA), carry increment adder (CIA), and carry select adder (CSLA) have been proposed for improving the speed of the RCA [2]. Another family of adders is parallel adders which, generally, have higher speeds and larger powers and area usages [18]. Most of the previous works on adders have focused on improving the speed and reducing the power and area usage of the adder structure in a family by invoking circuit and architectural modifications (see, e.g., [9]-[16]). In this section, we briefly review some of the works related to the comparative study of the adders which have focused on the performance and reliability parameters.

Some techniques to estimate the power consumption and delay of the adders have been proposed in [17] and [18]. These techniques are fast and do not need extensive simulations by computer-aided design tools. The techniques do not consider the impacts of the spatial and temporal variations. Since the accuracies of these methods are low, they have not been used in this study. In [19], a method for designing (ultra-) low power arithmetic units has been proposed. For this purpose, similar to our work, an exploration on delay-energy space of the different adder structures (by varying the supply voltage) is performed. The authors, however, have not investigates the impact of the supply voltage reduction on the speed and reliability of the adder structures. A framework for decreasing the delay and power consumption of the carry look-ahead adders (CLAs) has been proposed in [20]. The framework enabled the delay reduction under an energy consumption constraint. The proposed framework performed an exploration for different tree structures of the CLAs and logic styles for optimizing the energy-delay product (EDP) of the adder. The proposed framework performed the exploration at the nominal supply voltage and did not consider the impacts of different types of the variations on the reliability of the adders.

In [21], some energy-efficient design methodologies for designing high performance adder structures were proposed. The methodologies explore among different architectures, different logic families (e.g., static CMOS and dynamic CMOS) and different circuit-levels techniques (e.g., gate sizing) to reduce the delay and energy consumptions of the adders. In [22] and [23], circuit-level methods to optimize the parameters of the full adders (FAs) structures were proposed. In these works, the speed, power/energy consumption, and the number of the transistors in the FAs under different logic families were optimized. In [24] and [25], in addition to the delay, energy, and area, the reliabilities of the FA families in the presence of the process variation have been investigated. Note that the impact of the aging on the FAs was not considered in these works. In [26] and [27], the impact of reducing the supply voltage down to the near-threshold region on the performance of the FAs has been investigated and some methods to improve the performance have been proposed. Also, the performance of a 1-bit conventional static FA realized using the FinFET technology at near-threshold region has been investigated in [27].

In [28]-[34], some variable-latency adder structures have been proposed to reduce the power consumption and increasing the reliability of the adders without a considerable change of the nominal speed. In these works, the conventional structures were modified and/or some hybrid structures based on the 
TABLE I SUMmary OF THE Works DEALING WITH THE COMPARISON OF THE PERFORMANCE PARAMETERS OF THE AdDER STRUCTURES

\begin{tabular}{|c|c|c|c|c|c|c|c|c|c|c|c|c|c|c|c|}
\hline \multirow{2}{*}{ Work } & \multicolumn{2}{|c|}{ Structure } & \multicolumn{2}{|c|}{ Technology } & \multirow{2}{*}{ Delay } & \multirow{2}{*}{ Power } & \multirow{2}{*}{ Energy } & \multirow{2}{*}{ EDP } & \multirow{2}{*}{ Area } & \multirow{2}{*}{$\begin{array}{c}\text { Process } \\
\text { Variation }\end{array}$} & \multirow{2}{*}{$\begin{array}{l}\text { NBTI } \\
\text { Aging }\end{array}$} & \multirow{2}{*}{$\begin{array}{l}\text { STV } \\
\text { Region }\end{array}$} & \multirow{2}{*}{$\begin{array}{l}\text { NTV } \\
\text { Region }\end{array}$} & \multirow{2}{*}{$\begin{array}{l}\text { Nominal } \\
V_{D D}\end{array}$} & \multirow{2}{*}{$\begin{array}{c}\text { Focused on } \\
\text { Comparative } \\
\text { Study }\end{array}$} \\
\hline & CPAs & PPAs & Bulk & FinFET & & & & & & & & & & & \\
\hline [17] & $\bar{x}$ & $\checkmark$ & $\checkmark$ & $\bar{x}$ & $\checkmark$ & $\checkmark$ & $\checkmark$ & $x$ & $x$ & $x$ & $x$ & $x$ & $x$ & $\checkmark$ & $x$ \\
\hline [18] & $x$ & $\checkmark$ & $\checkmark$ & $x$ & $\checkmark$ & $\checkmark$ & $\checkmark$ & $x$ & $x$ & $x$ & $x$ & $x$ & $x$ & $\checkmark$ & $x$ \\
\hline [19] & $\checkmark$ & $\checkmark$ & $\checkmark$ & $x$ & $\checkmark$ & $\checkmark$ & $\checkmark$ & $\checkmark$ & $x$ & $x$ & $x$ & $\checkmark$ & $x$ & $\checkmark$ & $x$ \\
\hline [20] & $x$ & $\checkmark$ & $\checkmark$ & $x$ & $\checkmark$ & $\checkmark$ & $\checkmark$ & $x$ & $x$ & $x$ & $x$ & $x$ & $x$ & $\checkmark$ & $x$ \\
\hline$[21]$ & $x$ & $\checkmark$ & $\checkmark$ & $x$ & $\checkmark$ & $\checkmark$ & $\checkmark$ & $x$ & $x$ & $x$ & $x$ & $x$ & $x$ & $\checkmark$ & $x$ \\
\hline [35] & $\checkmark *$ & $\checkmark$ & $\checkmark$ & $x$ & $x$ & $x$ & $x$ & $x$ & $x$ & $x$ & $\checkmark$ & $x$ & $x$ & $\checkmark$ & $\checkmark$ \\
\hline [36] & $\checkmark *$ & $\checkmark$ & $x$ & $\checkmark$ & $x$ & $x$ & $x$ & $x$ & $x$ & $x$ & $\checkmark$ & $x$ & $x$ & $\checkmark$ & $\checkmark$ \\
\hline [37] & $\checkmark$ & $\checkmark$ & $\checkmark$ & $x$ & $x$ & $x$ & $x$ & $x$ & $x$ & $x$ & $\checkmark$ & $x$ & $x$ & $\checkmark$ & $\checkmark$ \\
\hline $\begin{array}{c}\text { Our } \\
\text { Work }\end{array}$ & $\checkmark$ & $\checkmark$ & $\checkmark$ & $\checkmark$ & $\checkmark$ & $\checkmark$ & $\checkmark$ & $\checkmark$ & $\checkmark$ & $\checkmark$ & $\checkmark$ & $\checkmark$ & $\checkmark$ & $\checkmark$ & $\checkmark$ \\
\hline
\end{tabular}

STV: SuperThreshold Voltage;

NTV: Neat-Threshold Voltage;

*: Only RCA structure;

variable latency operation were suggested. The structures led to increasing the performance and yield of the adders [3] and reduced the impact of the NBTI in nanometer designs. In [35], a study on the NBTI induced performance degradation of 32bit adders was presented. The study was only performed on RCA and some parallel adder structures. Furthermore, the study was performed only for the nominal supply voltage level while the effect of the voltage scaling on the performance of the adders and also the impact of the process variation on the performance of the adders were not considered. The studies performed in [35]-[37] on the effect of the NBTI on the CMOS binary adders have shown the importance of the reliability and performance of the adders in nanometers technologies.

None of these works has performed a complete study on the impacts of the aggressive voltage scaling including operating in the near-threshold region on the performance and reliability (i.e., speed, power/energy consumption, reliability under the process variations and aging) of the adders in the nano-scaled technologies. In this work, we perform this study which can help the designers to select a proper adder structure based on their considered design parameters. Table I compares some of the works focusing on the comparison of the adder structures or containing some discussions about comparing the adders. As the table indicates, the comparative study presented in this work includes more parameters from several aspects compared to those of these works. In the next section, we briefly describe the adder structures considered in this paper.

\section{CONSIDERED AdDER STRUCTURES}

For the comparative study performed here, four adder structures from carry propagate adder (CPA) and three adder structures from parallel prefix adders (PPAs) families were selected. The selected CPAs were RCA, CIA, CSKA, and CSLA, and the selected PPAs were Kogge-Stone adder (KSA) [38], Brent-Kong adder (BKA) [39], and Sklansky adder (SKA) [40]. These adders were selected based on the trade-off between speed, power/energy consumption, area usage, and also wiring and complexity of their layouts [2] [41]. Next, the internal structures and characteristics of the selected adders are discussed briefly here for the sake of being self-contained while one may find more details about them in [1] and [2].

\section{A. CPA Structures}

The internal structure of the RCA which includes a chain of FAs is shown in Fig. 2(a). The critical path delay is proportional to the $N$ where $N$ is the length of the input bits [2]. In the case of CIA, there are two levels of adders where, the first level contains $N / M$ blocks of $M$-bit RCA and the second level includes incremental adder blocks which calculate the final result [1]. The incremental adders may be implemented by a chain of half adders (HAs) which is depicted in Fig. 2(b). Compared to the RCA structure, the area usage, power consumption, and wiring of the CIA is larger than those of the RCA while its delay is smaller. It should be noted that in this work, the optimum value of $M$ was determined based on the selected technologies [2]. The structure of an $N$-bit CSKA, which is based on the RCA blocks, is shown in Fig. 2(c). In addition to the chain of FAs in each stage, there is a carry skip logic (2:1 multiplexer). In the case where a group of cascaded FAs are in the propagate mode, the carry output of the chain is equal to the carry input. In the CSKA, the carry skip logic detects this situation, and makes the carry ready for the next stage without waiting for the operation of the FA chain to be completed. The skip operation is implemented using the gates and the multiplexer shown in the Fig. 2(c). CSKA may be implemented using fixed or variable block sizes (FBS and VBS) where the highest speed may be obtained for the VBS structure [42] [43]. In this case, the block size is the same as that of the RCA block. The critical path delay of the CKSA is much smaller than the one in the RCA, while having about the same area and power as those of the RCA [2]. In this work, the variable block size CSKA (VBS-CSKA) is considered where for each technology, the optimum size for each block is considered [43].

The internal structure of the $N$-bit CSLA is shown in Fig. 2(d) where the first stage is an RCA block with $C_{i n}$ as the input carry, and the other stages contain two RCA blocks which calculate the partial sums and output carry of the stage when the $C_{i n}=1$ and $C_{i n}=0$. In the next level, using multiplexers the final sums and output carry are selected based on the actual carries. There are two approaches to implement the CSLA which are linear and square-root approaches [2]. In the linear approach, the size of the RCA blocks in all the stages 


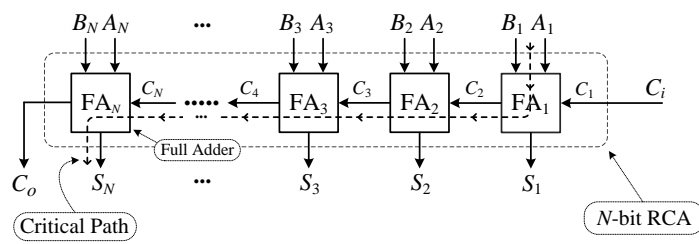

(a)

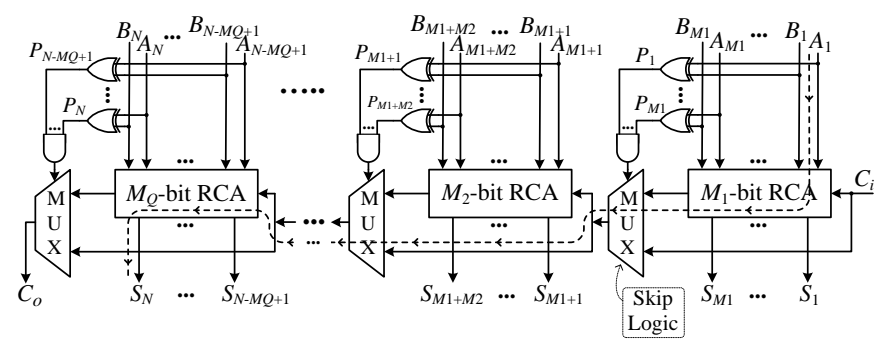

(c)

Fig. 2. Internal structures of a) RCA, b) CIA, c) CSKA, and d) CSLA [1] [2].

are the same, while in the square-root approach, the size of the RCA blocks from the first stage to the last stage are increased. The speed of the square-root CSLA (SQRT-CSLA) is higher than that of the linear one [2] [44], and hence, in this work, the SQRT-CSLA is chosen for the proposed investigation. It should be noted in this work, the optimal implementation (i.e., selecting the best size of the RCA block in each stage) of the SQRT-CSLA is determined based on [44]. The critical paths of these structures are indicated by dashed lines in Fig. 2.

\section{B. PPA Structures}

The parallel prefix adders which are also called look-ahead use the direct parallel prefix scheme for a fast carry computation [2]. The overall structure of a PPA is shown in Fig. 3(a) which contains three stages. These stages are preprocessing stage, parallel prefix network, and postprocessing stage. In the preprocessing stage, the propagate $(P)$ and generate $(G)$ signals are calculated in parallel for each input bit position. These signals are used in the parallel prefix network to generate the intermediate carries. Finally, in the last stage, i.e., post-processing stage, the final sum and output carry are calculated using the intermediate carries and propagate signals. There are different types of the parallel prefix algorithms that lead to different PPA structures with different performances. All of the PPA structures have the preprocessing and post-processing stages, and differ only in the parallel prefix network for generating intermediate carries. Parallel prefix networks may be classified with a threedimensional taxonomy corresponding to the number of logic levels, fan-out, and wiring tracks [41]. The Kogge-Stone (KS), Brent-Kung (BK), and Sklansky (SK) networks occupy the vertices of this three-dimensional taxonomy. These networks, in the case of $N=16$, are shown in Fig. 3(b)-(d), respectively, where the critical paths are indicated by dashed lines. The functionality of the black and gray sub-blocks and also other sub-blocks in these structures are illustrated in Fig. 3(e).

The logic levels of the KS and SK parallel prefix networks,

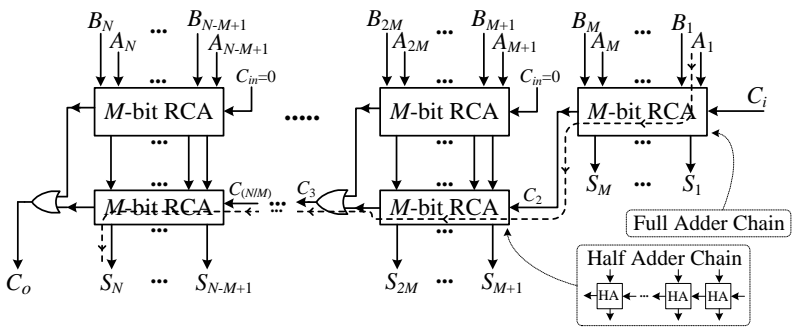

(b)

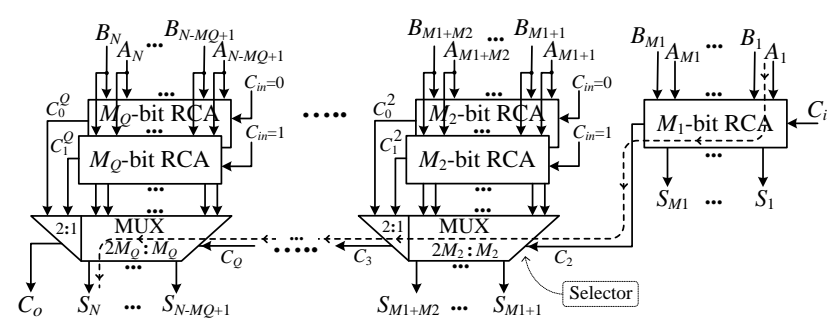

(d)

which are similar to each other, are smaller than that of the BK parallel prefix network. Also, the KS and BK parallel prefix networks have similar fan-outs which are almost smaller than that of the SK parallel prefix network. Additionally, the KS has the largest wiring tracks while the wiring tracks of the BK and SK parallel prefix networks are almost similar to each other. The speed of the KSA (BKA) is the highest (smaller) while its area usage and power consumption are higher (smaller) than the other two adders [41]. It should be noted that the structure complexities of PPAs are more than those of the other adder schemes (CPAs) [1] [2]. Since these networks have different properties, we have selected them in our comparative study. Finally, Table II summarizes the design characteristics and implementation details for each adder architecture such as area, delay, logic levels, maximum (Max) fan-out, wiring complexity, and structure regularity (synthesis) which $N$ is the bit-length of adder structures.

In this work, the 32-bit structures of the adders (i.e., CPAs and PPAs) at the gate level (see Fig. 2 and Fig. 3) were synthesized automatically by the Synopsys Design Compiler tool [65] under the high speed constraint using the (typical) NanGate standard cell libraries for both the bulk CMOS [46] and FinFET [66] technologies. Therefore, based on this constraint, the sizes of the standard cells for each adder structure in each technology were determined and the results presented in this work are for this case. It should be noted that the standard cells used for the 45-nm bulk and 14-nm FinFET technologies were from the NanGate open cell library. Also, this library has the standard cells for the 15-nm FinFET library [66] and since it does not have its transistor model required for the HSPICE simulations, we have used the 14-nm FinFET transistor model of the PTM [45]. Additionally, due to the lack of interconnect SPICE model for these two technologies, the simulations were performed without considering them. Since the interconnect Macro Models of these two technologies for the Cadence SOC Encounter [47] exist, this tool was used for estimating the areas of the adders. 


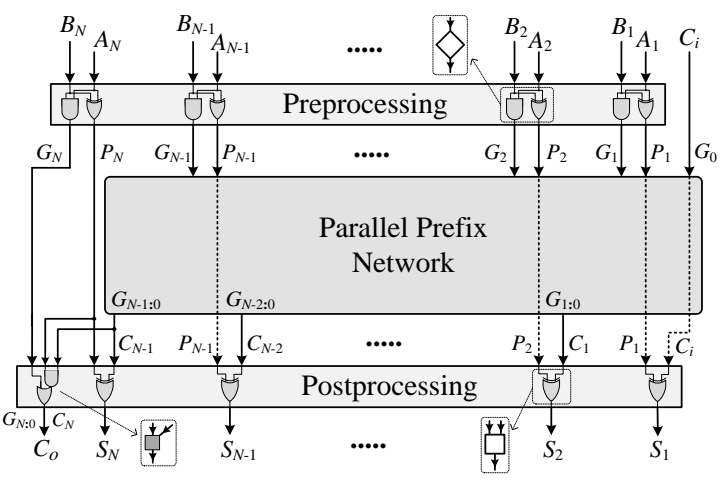

(a)

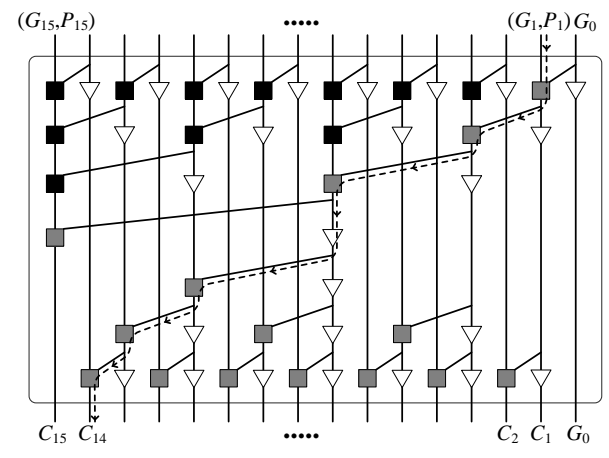

(c)

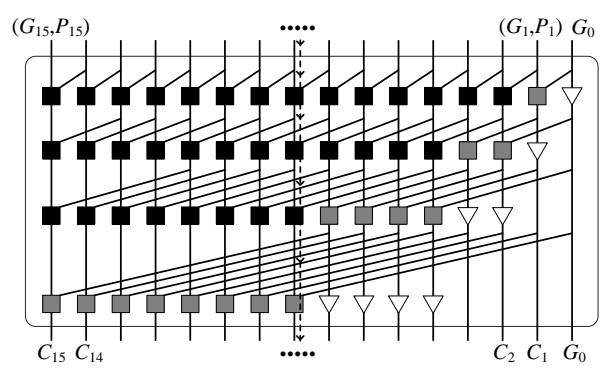

(b)

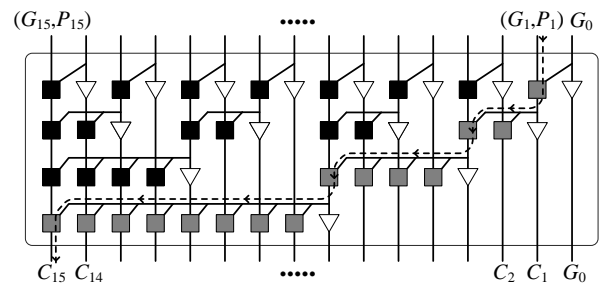

(d)

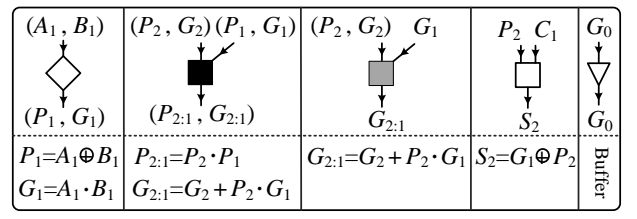

(e)

Fig. 3. (a) Overall structure of the parallel prefix adders (PPAs). (b) KS, (c) BK, and (d) SK parallel prefix networks. (e) Functionality of the different subblocks. Three parallel prefix networks are depicted for $N=16$ [41].

TABLE II CLASSIFICATION OF THE AdDER ARCHITECTURES [2]

\begin{tabular}{|c|c|c|c|c|c|c|c|c|}
\hline \multicolumn{2}{|c|}{$\begin{array}{c}\text { Adder } \\
\text { Architecture }\end{array}$} & Area & Delay & $\begin{array}{c}\text { Area-Delay } \\
\text { Product }\end{array}$ & $\begin{array}{l}\text { Logic } \\
\text { Levels }\end{array}$ & $\begin{array}{c}\text { Maximum } \\
\text { Fan-out }\end{array}$ & $\begin{array}{c}\text { Wiring } \\
\text { Complexity }\end{array}$ & $\begin{array}{r}\text { Structure } \\
\text { Regularity }\end{array}$ \\
\hline \multirow{4}{*}{$\sum_{0}^{n}$} & RCA & $O(N)$ & $O(N)$ & $O\left(N^{2}\right)$ & $O(N)$ & 1 & lowest & highest \\
\hline & CIA & $O(N)$ & $O(\sqrt{N})$ & $O(N \sqrt{N})$ & $O(\sqrt{N})$ & 1 & low & high \\
\hline & VBS-CSKA & $O(N)$ & $O(\sqrt{N})$ & $O(N \sqrt{N})$ & $O(\sqrt{N})$ & 2 & medium & high \\
\hline & SQRT-CSLA & $O(N)$ & $O(\sqrt{N})$ & $O(N \sqrt{N})$ & $O(\sqrt{N})$ & $O(\sqrt{N})$ & medium & high \\
\hline \multirow{3}{*}{$\sum_{1}^{\infty}$} & KSA & $O(N \log N)$ & $O(\log N)$ & $O\left(N \log ^{2} N\right)$ & $\log N+2$ & 2 & highest & medium \\
\hline & BKA & $O(N \log N)$ & $O(\log N)$ & $O\left(N \log ^{2} N\right)$ & $2 \log N+1$ & 2 & medium & medium \\
\hline & SKA & $O(N \log N)$ & $O(\log N)$ & $O\left(N \log ^{2} N\right)$ & $\log N+2$ & $N / 2+1$ & high & medium \\
\hline
\end{tabular}

TABLE III

AREA USAGES AND NUMBERS OF TRANSISTORS OF THE ADDERS

\begin{tabular}{|l|c|c|c|}
\hline \multirow{2}{*}{$\begin{array}{c}\text { 32-bit Adder } \\
\text { Structure }\end{array}$} & \multicolumn{2}{|c|}{ Area $\left(\mu \mathrm{m}^{2}\right)$} & \multirow{2}{*}{$\begin{array}{c}\text { of } \\
\text { Transistors }\end{array}$} \\
\cline { 2 - 3 } & 45 -nm Bulk & 14-nm FinFET & 896 \\
\hline RCA & 151.3 & 42.0 & 1402 \\
\hline CIA & 230.0 & 59.9 & 1464 \\
\hline VBS-CSKA & 253.1 & 71.8 & 2096 \\
\hline SQRT-CSLA & 357.7 & 103.3 & 2010 \\
\hline KSA & 403.2 & 108.2 & 1402 \\
\hline BKA & 286.1 & 77.8 & 1668 \\
\hline SKA & 328.7 & 92.2 & \\
\hline
\end{tabular}

Table III reports the area usage and the number of transistors for each adder structure in the 45-nm bulk and 14nm FinFET technologies. The RCA has the smallest area while the KSA has the highest area. The next largest adder is
SQRT-CSLA which its area is about 1.25 times larger than that of the BKA and slightly larger than that of the SKA. Also, SQRT-CSLA has the highest number of transistors. The areas of the CIA and VBS-CSKA structures are almost similar and are higher than that of the RCA.

\section{ImPaCt OF Voltage SCALING ON THE PERFORMANCE OF ADDERS}

In this section, different performance parameters of the adder structures versus the supply voltage are compared. The comparison includes the delay, power, and energy in both superthreshold and near-threshold regions of operation. The 32-bit structures of the adders were simulated using HSPICE tool [48] for both the bulk and FinFET technologies. The nominal supply voltage of the bulk (FinFET) technology was $1.1 \mathrm{~V}(0.8 \mathrm{~V})$ and the threshold voltages of the nMOS (nFET) and pMOS (pFET) transistors were $0.67 \mathrm{~V}(0.4 \mathrm{~V})$ and $-0.62 \mathrm{~V}$ 


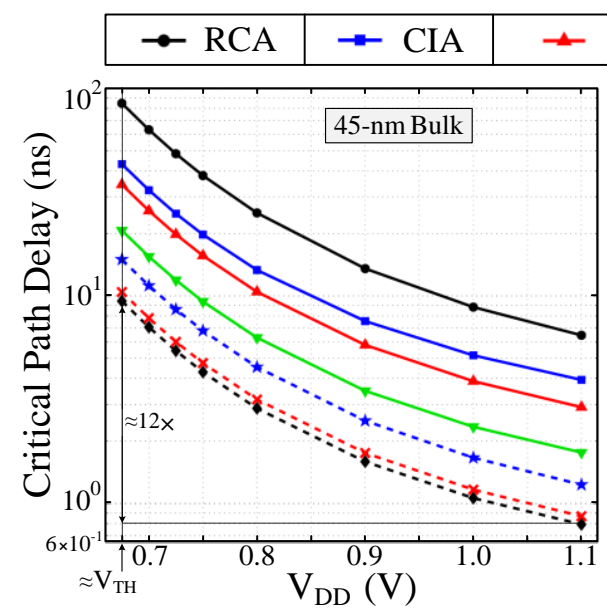

(a)

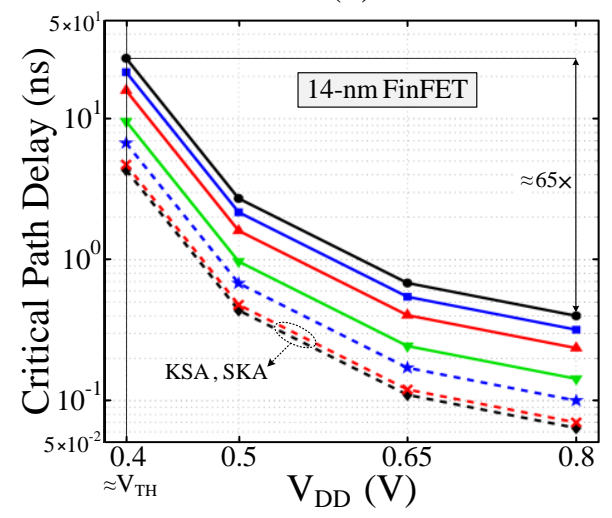

(d)

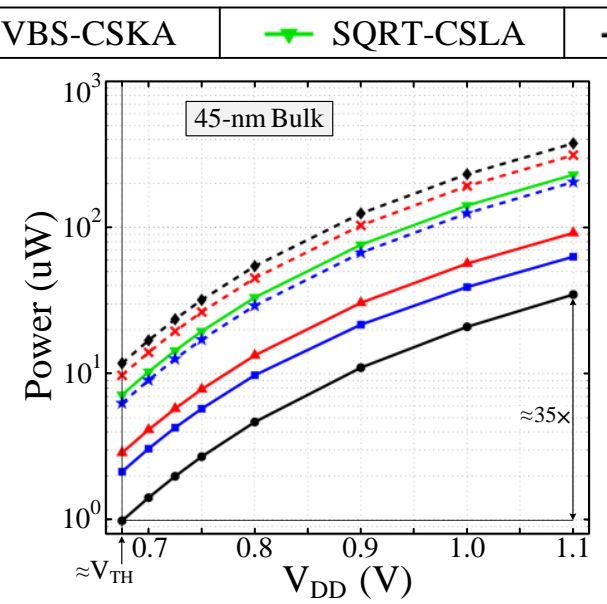

(b)

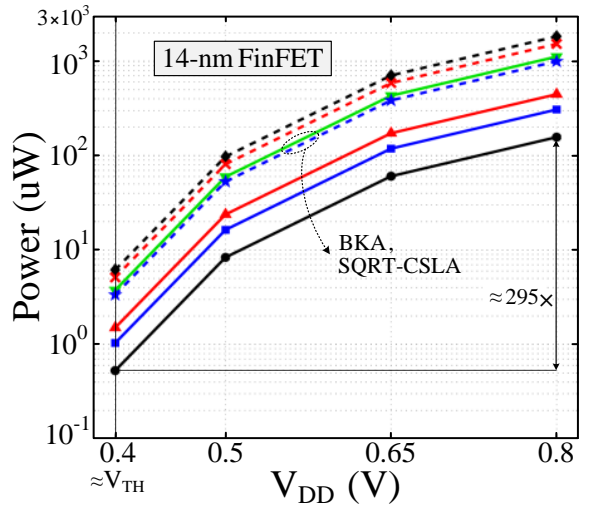

(e)

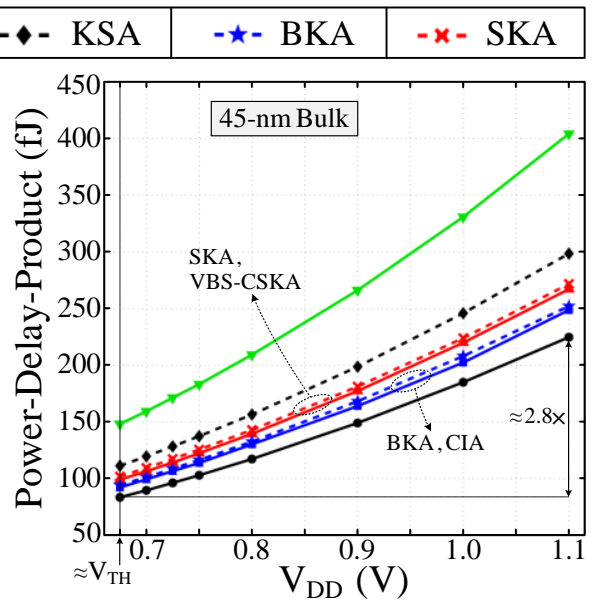

(c)

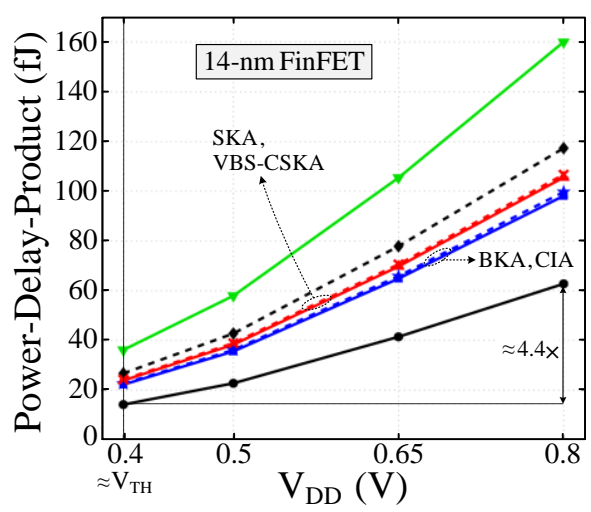

(f)

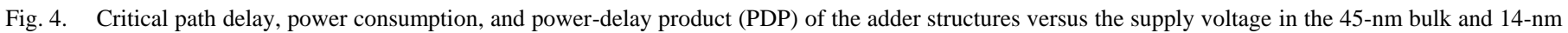
FinFET technologies.

$(-0.4 \mathrm{~V})$, respectively. All the studies were performed under the room temperature of $25{ }^{\circ} \mathrm{C}$. It should be noted that, to extract the power consumption of the adders, 10,000 uniform random stimuli were applied to the adders. The input injection rates were chosen based on the maximum operating frequency of the adder structure at each supply voltage.

First, the critical path delays of the adder structures versus the supply voltage are plotted in Fig. 4(a) and (d) for the 45$\mathrm{nm}$ bulk and 14-nm FinFET technologies, respectively. In general, the delay increases for all the adders almost similarly by decreasing the supply voltage. As was expected, the three PPA structures have smaller delays compared to those of the four CPA structures, due to the parallel structure of the PPAs. Also, the RCA (KSA) has the highest (lowest) delay due to its serial (parallel) structure at all supply voltages. Also, the critical path delay of the CIA is smaller than that of the RCA. Also, the delay of the VBS-CSKA is smaller than those of the CIA and RCA due to the carry skipping property of the structure. The SQRT-CSLA has the smallest delay compared to the other studied CPAs which is due to the logic duplication in its structure. Among the PPA structures, the highest delay at all the voltages belongs to the BKA which is due to the large logic level of its parallel prefix network. On the other hand, the KSA structure, because of its smallest logic level and small fan-out in its parallel prefix network, has the smallest delay. The SKA has the higher fan-out in its parallel prefix network, and hence, its delay is slightly larger than that of the KSA. When comparing the two technologies, it is observed that the differences between the delays of the adders in the 14$\mathrm{nm}$ FinFET are changed compared to those of the corresponding adders in the $45-\mathrm{nm}$ bulk technology. This may be attributed to the performance features of different logic resources used in each adder architecture in the corresponding technology node. Finally, the results indicate that reducing the supply voltage from $1.1 \mathrm{~V}(0.8 \mathrm{~V})$ (nominal voltage) to the near-threshold voltage causes an about 12 (65) fold increase in the delay for all the adders implemented in the 45-nm bulk (14-nm FinFET) technology. The higher delay increase in the 14-nm FinFET by the voltage scaling is owing to the stronger gate control of the channel in this technology as compared to the conventional one [49] [50].

The power consumptions of the adders versus the supply voltage are shown in Fig. 4(b) and (e) for the 45-nm bulk and 14-nm FinFET technologies, respectively. The results indicate that the three PPAs and SQRT-CSLA structures have larger power consumptions compared to the RCA, CIA, and VBSCSKA structures at all voltages. This may be attributed to the parallel structure of the PPAs and logic duplication in the 


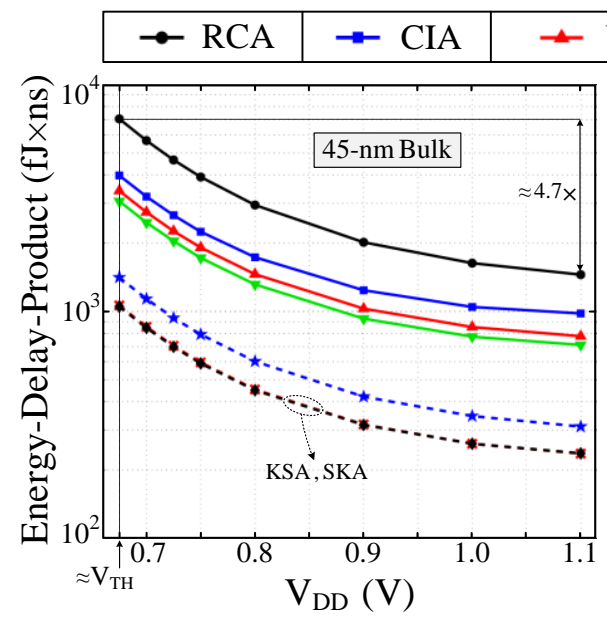

(a)

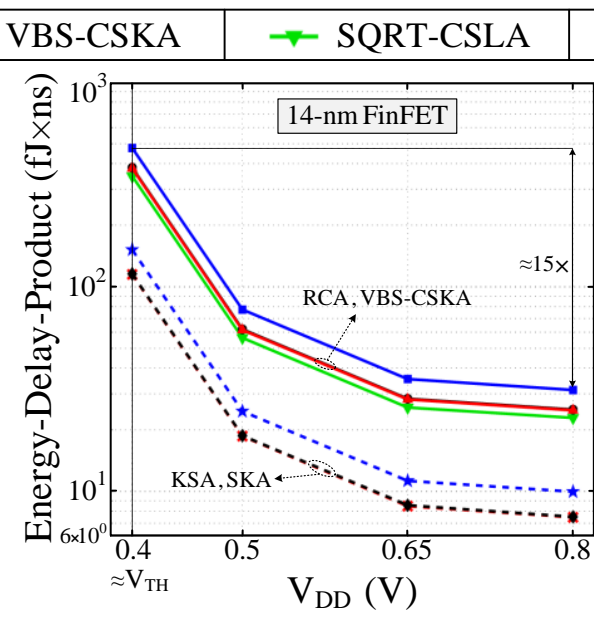

(b)

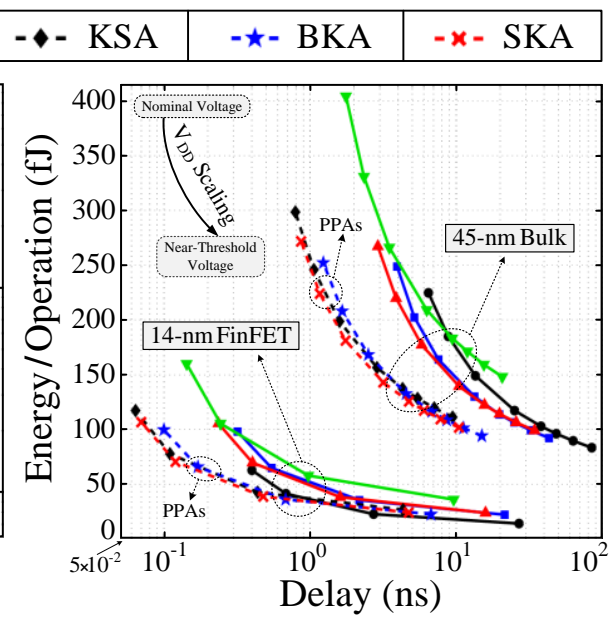

(c)

Fig. 5. Energy-delay product (EDP) of the adder structures versus the supply voltage for the (a) 45-nm bulk CMOS, and (b) 14-nm FinFET technologies. (c) Energy-delay pareto-optimal curves of the adder structures for both 45-nm bulk CMOS and 14-nm FinFET technologies.

SQRT-CSLA. The smallest power consumption belongs to the RCA owing to its simple and regular structure while the KSA structure consumes the highest power owing to its parallel structure and dense wiring tracks. The power consumption of the SKA is smaller than that of the KSA which is due to the smaller sub-blocks and wiring tracks. Also, the BKA has the smallest power consumption among the PPAs which originates from the fact that its parallel prefix network is sparse. It should be noted that the power consumption of the SQRT-CSLA is almost the same as that of the BKA. As mentioned before, the high power consumption of the SQRTCSLA is due the duplicated blocks in its structure. As shown in Fig. 4(b) and (e), the trend of decreasing the power consumption by decreasing the supply voltage is almost similar for all the adders, and the results reveal, on average, a 35 (295) times reduction in the power consumption of the adders implemented in the 45-nm bulk (14-nm FinFET) technology when scaling the supply voltage from $1.1 \mathrm{~V}$ $(0.8 \mathrm{~V})$ to the near-threshold voltage. Also, the considerable difference between the power reductions of the adder structures due the voltage reduction in the two technologies is again attributed to the strong gate control of the channel in the case of the FinFET technology [49] [50].

Fig. 4(c) and (f) show the power-delay product (PDP) of the adders at different supply voltage levels for the 45-nm bulk and 14-nm FinFET technologies, respectively. The RCA has the best PDP compared to those of the other structures in the supply voltage range considered in this work. This is due to the smallest power consumption of this structure. The worst PDP belongs to the SQRT-CSLA with that of the KSA is next to it. The PDP of the SQRT-CSLA is about 1.4 (1.3) times more than that of the KSA structure in the 45-nm bulk (14-nm FinFET) technology, due to the higher power and smaller speed of the SQRT-CSLA structure compared to those of the KSA. Additionally, the PDPs of the CIA and VBS-CSKA are almost similar to the PDPs of the BKS and SKA, respectively, in the both considered technologies. Furthermore, the PDPs of these four adders are larger than the PDP of RCA and smaller than the PDP of KSA. Consequently, among PPAs, BKA with the smallest power consumption and PDP and with an acceptable speed is a proper adder in high performance and low power applications. The SQRT-CSLA, due to the large PDP and delay (compared to those of the PPAs), is not a proper choice in high performance and low power applications. Also, the results show that the trends of the PDP changes by the voltage scaling are almost similar for the adders. Finally, note that scaling the supply voltage from 1.1 $\mathrm{V}(0.8 \mathrm{~V})$ to the near-threshold voltage leads to, on average, about 2.8 (4.4) times reduction in the PDP of the adders implemented in the 45-nm bulk (14-nm FinFET) technology.

Fig. 5(a) and (b) show the energy-delay products (EDPs) of the adders versus the supply voltage for the 45 -nm bulk and 14-nm FinFET technologies, respectively. As the results shown in Fig. 5(a), the high delay of the RCA causes the largest EDP for this structure among other adders in 45-nm bulk technology. In the case of the FinFET technology, the higher power consumption of the CIA structure and lower delay difference with that of the RCA, bring up the largest EDP for the CIA in 14-nm FinFET technology. The EDPs of the KSA and the SKA are almost similar and are the smallest EDPs among those of the other adders. Generally, the EDPs of the PPAs are smaller than those of the CPAs. Among the PPAs (CPAs), the BKA (SQRT-CSLA) has the largest (smallest) EDP due to its highest (smallest) delay. The EDP of the VBS-CSKA structure is smaller (larger) than that of the CIA (SQRT-CSLA) in the 45-nm bulk technology while EDP of this structure is almost the same as that of the RCA in the 14-nm FinFET technology. The results show that reducing supply voltage from nominal voltage to near-threshold voltage leads to, on average, about 4.7 (15) fold increase in the EDP for all the adders implemented in the $45-\mathrm{nm}$ bulk (14-nm FinFET) technology.

Finally, to demonstrate the trade-offs between the delay and energy for each adder structure, the energy-delay paretooptimal curves are plotted in Fig. 5(c) which reveal that the PPAs have better trade-offs between the energy and delay 


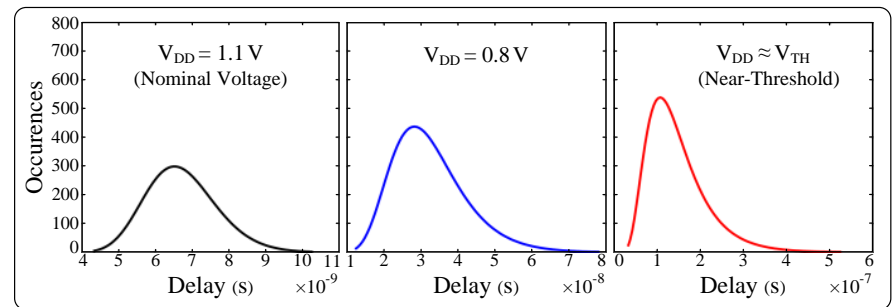

(a)

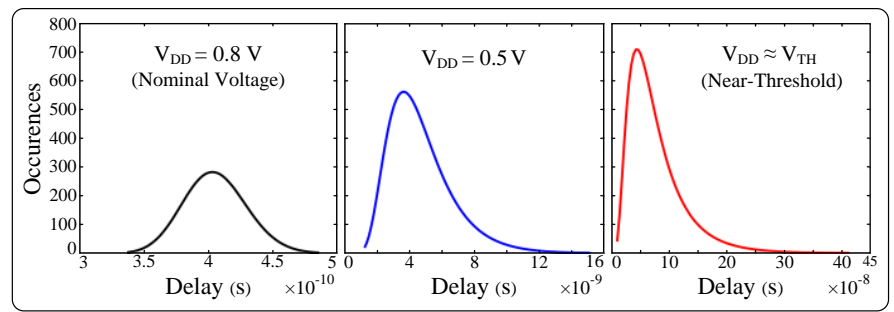

(c)

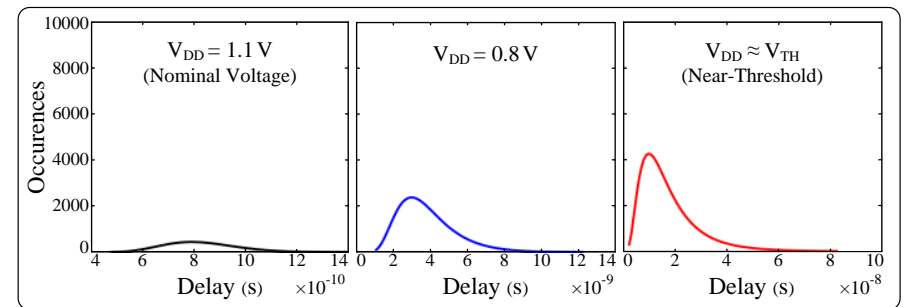

(b)

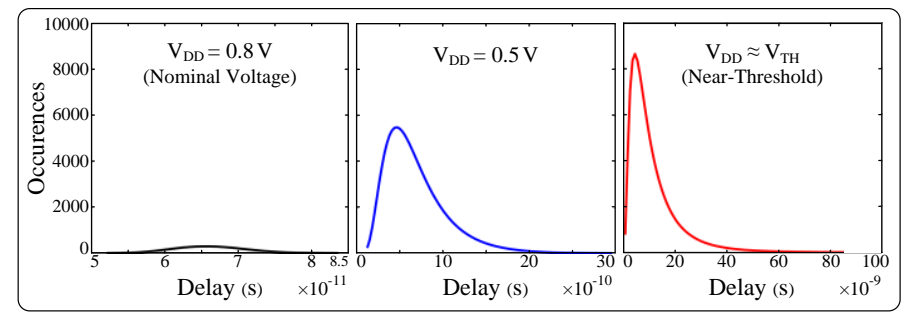

(d)

Fig. 6. Delay distributions of the (a) RCA for 45-nm bulk technology, (b) KSA for 45-nm bulk technology, (c) RCA for 14-nm FinFET technology, and (d) KSA for 14-nm FinFET technology under the different supply voltage levels.

compared to those of the CPA structures. Also, among the CPAs, the VBS-CSKA has the best trade-offs between the delay and energy while in the near-threshold region, the tradeoffs between the delay and energy of the CIA becomes almost similar to that of the VBS-CSKA. The trade-off between the delay and energy of the RCA in the superthreshold is better than the trade-off in the case of the SQRT-CSLA in the nearthreshold region. It means that instead of using CSLA-SQRT in the near-threshold operating region, one may use RCA in the superthreshold operating region where the area usage of the RCA is considerably smaller. Also, the SKA structure has the best trade-off between the delay and energy in the superthreshold operating region while by decreasing the supply voltage, the trade-offs between the delay and energy of the all PPA structures become almost similar. Finally, it should be noted that, in the near-threshold voltage region, the energy-delay pareto-optimal curves of the PPAs become closer to those of the CPAs in 14-nm FinFET technology.

\section{IMPACT OF Voltage SCALING ON THE RELIABILITY OF ADDERS}

In this section, the impacts of the process variation on the time zero delay uncertainty and the NBTI aging on the delay degradation of the adders are studied.

\section{A. Impact of the Process Variation}

As was shown in Section IV, operating in the near-threshold region leads to reducing the power and energy consumption. In this region, however, due to the decrease in the supply voltage which leads to the decrease in the gate overdrive $\left(V_{D D}-V_{T H}\right.$, where $V_{D D}$ is the supply voltage and $V_{T H}$ is the threshold voltage), and hence, the ON current $\left(I_{\mathrm{ON}}\right)$, the impact of the process variation on the delay uncertainty is increased significantly [4] [7]. There are different sources of variations which among them, random dopant fluctuations (RDFs) are known to be the dominant factor of the $V_{T H}$ variation in the conventional bulks [5], while its impact is suppressed in FinFETs [51] [52]. In addition, line edge roughness (LER) is a significant factor for advanced technology nodes, which leads to the channel length $(L)$ as well as $V_{T H}$ variations of the nanoscale transistors [52]. In the FinFETs, due to the importance of the device geometry, the impact of LER (and also fin thickness $\left(t_{\text {fin }}\right)$ ) is considerably larger compared to that of the conventional bulks [51]-[54].

To evaluate the effect of the process variation on the performance uncertainty of the adders versus the supply voltage scaling, we have used the Monte-Carlo simulations using SPICE [48]. The two (three) dominant variation sources, $V_{T H}$ and $L\left(V_{T H}, L\right.$, and $\left.t_{\text {fin }}\right)$ of the nMOS (nFET) and pMOS (pFET) transistors are represented by normal distributions and inserted into the 45-nm bulk (14-nm FinFET) models. In this study, we have used the same simulation setups which were discussed in Section IV. Additionally, for the simulations, we have considered $10 \%(5 \%)$ and $5 \%(2.5 \%)$ local (global) variation $(\% \sigma / \mu)$ for $V_{T H}$ and $L$, respectively, in the 45 -nm bulk [55] [56]. Also, we have considered 5\% (2.5\%), 15\% (7.5\%), and $15 \%(7.5 \%)$ local (global) variation $(\% \sigma / \mu)$ for $V_{T H}, L$, and $t_{\text {fin }}$, respectively, in the 14-nm FinFET technology [51]-[54]. To extract the impact of the process variation, we have performed Monte-Carlo simulation under 10,000 samples for each supply voltage. It should be noted that the normal distributions were assumed for the $V_{T H}, L$, and $t_{\text {fin }}$ variations.

Fig. 6 shows the delay distributions of the RCA (slowest adder structure) and KSA (fastest adder structure) at the nominal voltage, near threshold voltage, and a voltage in between them for both technologies. The distributions appear to be normal (skewness $\approx 0$ ) in the superthreshold region. At lower supply voltages, the skewness of the distributions increase such that in the near-threshold region, the delay variations may be modeled by a log-normal distribution. It is worth mentioning that by decreasing the supply voltage to the near-threshold region, the skewness of the delay distribution is increased. As the distributions in Fig. 6 show, the delay variation of the KSA is more than that of the RCA. This shows that the impact of the process variation is a strong function of the adder structure. To study this further, we obtain the 


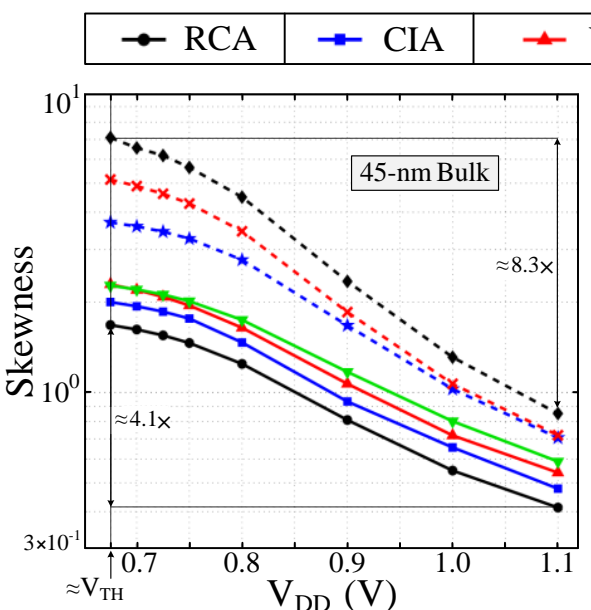

(a)

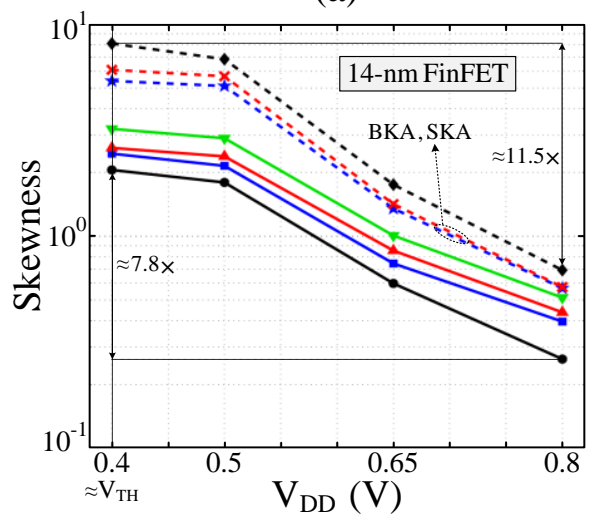

(d)

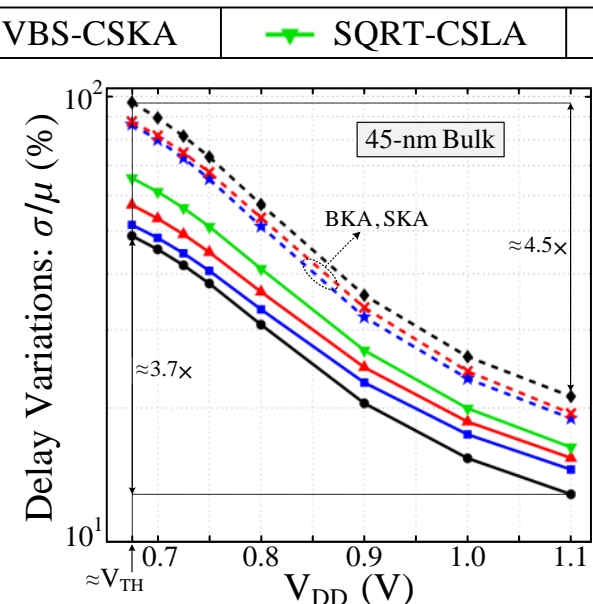

(b)

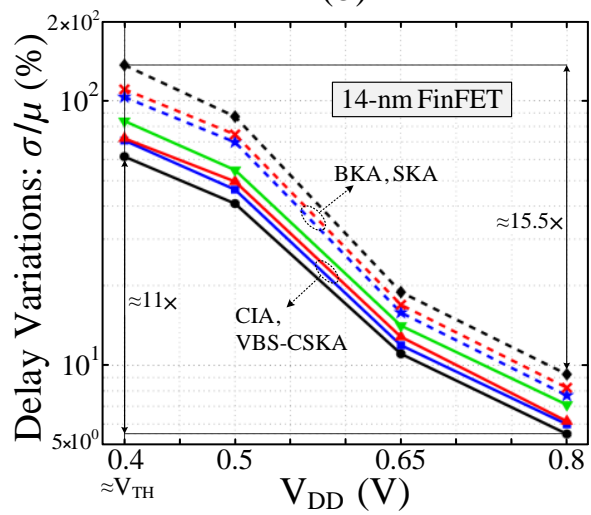

(e)

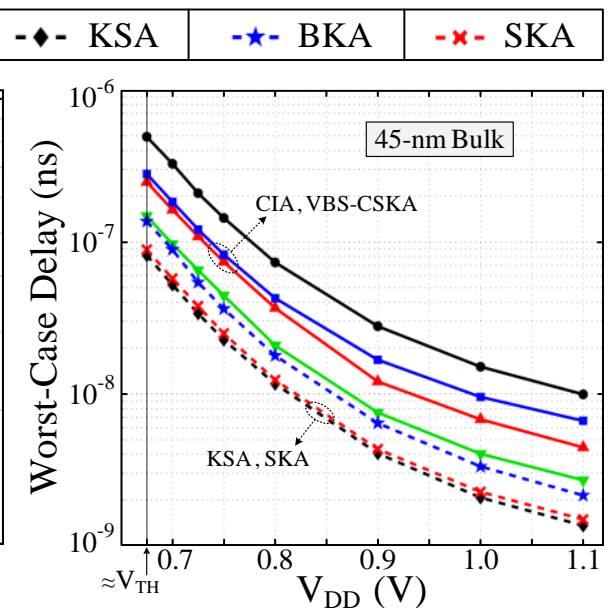

(c)

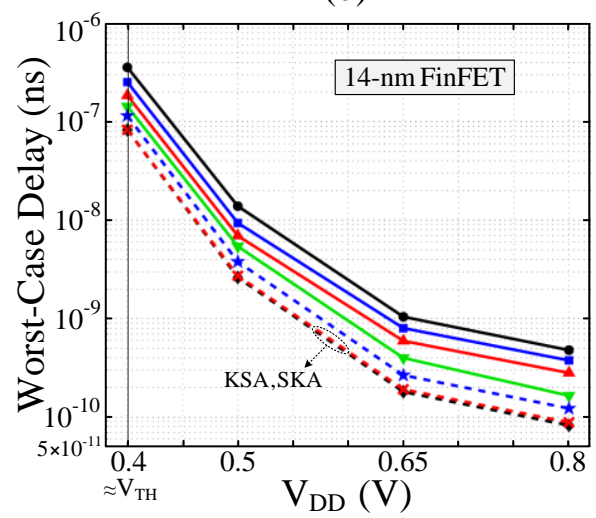

(f)

Fig. 7. Skewness of the delay distributions, delay variations $(\% \sigma / \mu)$, and worst-case delays of the adder structures versus the supply voltage for both 45 -nm bulk and 14-nm FinFET technologies in the presence of process variation.

skewness, delay variation, and worst case delay of the adders for both technologies as a function of the supply voltage.

Fig. 7(a) and (d) show the skewness of the delay distributions versus the supply voltage for the $45-\mathrm{nm}$ bulk and 14-nm FinFET technologies, respectively. As the results in these figures reveal, by reducing the supply voltage, the skewness of the delay distributions of the adders increase considerably where the KSA (RCA) adder has the highest (smallest) skewness for all the supply voltages. Furthermore, the amounts of the skewness for the CPA structures are smaller than those of the PPA structures. Next to the KSA, the SKA adder has the highest skewness which at high voltages, its skewness is close to that of the BKA. Among the CPAs, the SQRT-CSLA structure has the largest skewness. In the best case which belongs to the RCA structure, reducing the supply voltage from the nominal voltage to the near-threshold voltage causes about 4.1 (7.8) fold increase in the skewness of the delay distributions in the 45-nm bulk (14-nm FinFET) technology. Also, in the worst case which is for the KSA structure, reducing the voltage from the nominal voltage to the near-threshold voltage causes about 8.3 (11.5) fold increase in the skewness of the delay distribution in the 45-nm bulk (14$\mathrm{nm}$ FinFET) technology. It should be mentioned that the skewnesses of the delay distributions of the adders in 14-nm FinFET for the superthreshold voltage levels are smaller than those of the adders in the 45-nm bulk technology. However, for the near-threshold voltage levels, the skewnesses of the delay distributions of the adders in 14-nm FinFET are larger than those of the adders in the $45-\mathrm{nm}$ bulk, which originate from the higher increase in the skewness of the adders under the supply voltage reduction in the 14-nm FinFET (due to the strong gate control on the channel of FinFETs) compared to those of the adders in the 45-nm bulk technology.

Fig. 7(b) and (e) show the delay variations $(\% \sigma / \mu)$ of the adders versus the supply voltage for the 45 -nm bulk and 14nm FinFET technologies, respectively. As was expected, the supply voltage decrease leads to the considerable increase in the delay variations [4]. Note that the delay variations of the PPAs are more than those of the CPAs in the whole supply voltage range. In the worst case which is related to the KSA, the delay variation is about $4.5 \mathrm{x}(15.5 \mathrm{x})$, while in the best-case which belongs to the RCA, the delay variation is about $3.7 \mathrm{x}$ (11x) in the 45-nm bulk (14-nm FinFET) technology. The delay variations of the BKA and SKA structures are almost similar to each other in all supply voltage levels. Among the CPAs, SQRT-CSLA has the largest delay variation. Also, the increases in the delay variations of the CPAs by voltage reduction are smaller than those of the PPAs. The results show that in the PPA structures with fewer logic levels (LLs), the impact of the process variation is larger. Similarly, in the 


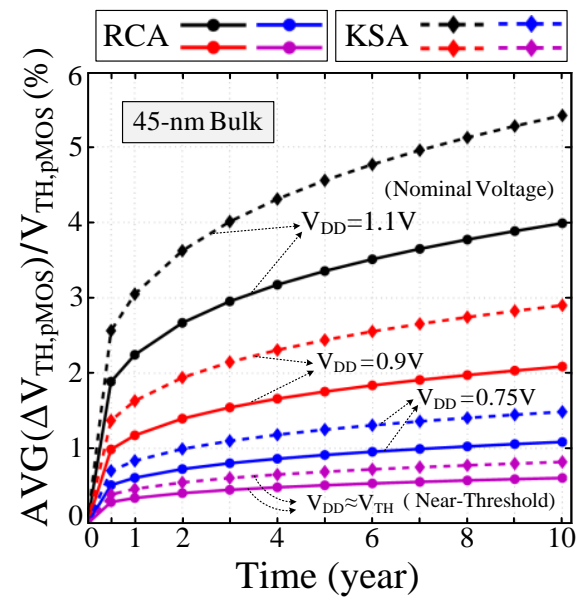

(a)

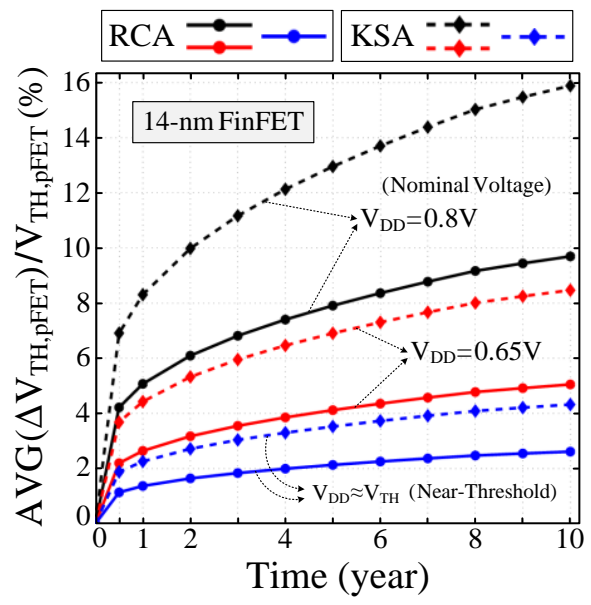

(b)

Fig. 8. NBTI induced threshold voltage degradation in the case of the RCA and KSA structures under the different supply voltage levels for the (a) 45-nm bulk and (b) 14-nm FinFET technologies.

adder structures with more logic levels (e.g., RCA and CIA), the delay variations are smaller. This claim is supported by the idea that the delay variation of a logic chain under the random fluctuation is inversely proportional to the square of the number of the logic gates in the chain [57]-[59]. This is translated to the lowest (highest) delay variation for the RCA (KSA) structure.

The worst case delays of the adders versus the supply voltage have been plotted in Fig. 7(c) and (f). The worst case delay is extracted from the delay distribution where the timing yield is at least $99.773 \%$ [59]. This corresponds to the point of $\mu+3 \sigma$ in the case of the normal distribution [59]. As the results show, the RCA (KSA) has the maximum (minimum) worst case delay among all the adders for the whole voltage range. It should be noted although KSA (RCA) had a large (small) delay variation, its worst case delay is the smallest (largest) compared to the other adders due to its small (large) nominal delay for this voltage range. As mentioned before, the delay variations of the PPAs are larger than those of the CPAs. However, due to the smaller delays of the PPAs, their worstcase delays are smaller than those of the CPAs. It is worth mentioning that, while the worst-case delays of all the structures increase when the supply voltage is decreased, the ratios of the worst-case delays of the adders in the nearthreshold region $\left(V_{D D} \approx V_{T H}\right)$ to their worst-case delays in the super-threshold region (nominal voltage) are different. These ratios for the cases of the RCA and KSA are about 49x (740x) and 60x $(1005 \mathrm{x})$ in the $45-\mathrm{nm}$ bulk (14-nm FinFET) technology, respectively.

\section{B. Impact of NBTI Aging on the Delay Degradation of Adders}

As mentioned before, temporal variations due to aging impacts on the reliability of the CMOS digital circuits during their lifetimes. One of the major causes of these variations is the negative-bias temperature instability (NBTI) phenomenon which is a key reliability issue in the MOSFET (both bulk and FinFET) technologies [5] [35]. The NBTI induces an increase in the threshold voltage and consequently decreases in the drain current of a pMOS (pFET) device. The degradation exhibits a logarithmic dependence on time [60] [61]. To model the NBTI aging impact on the adder structures, in this study, the MOS Reliability Analysis (MOSRA) package [62] [63] of the Synopsys HSPICE tool [48] was used. MOSRA models the NBTI degradation considering two phases of pre-stress and post-stress. During the pre-stress phase, the degradation in the threshold voltage $\left(\mathrm{V}_{\mathrm{TH}}\right)$ of all the pMOS (pFET) transistors in each adder structure is estimated based on their operating voltage, temperature, activity parameters, and device model parameters. The activity parameter is determined using the input patterns when applied continuously (as the adder workload) at different aging times. In our case, the 10,000 inputs which were obtained from a uniform random distribution were used. It should be noted that the threshold voltage shift for each transistor is determined separately. In the post-stress phase, the effect of NBTI aging on the worstcase delay of the adder at different points during the circuit lifetime is determined using the circuit degradation (i.e., the threshold voltage drifts of all the pMOS and pFET transistors of each adder structure) calculated during the pre-stress phase.

Fig. 8 shows the ratios of the average (AVG) of the threshold voltage increment of the all pMOS (pFET) transistors (e.g., $\mathrm{AVG}\left(\Delta \mathrm{V}_{\mathrm{TH}, \mathrm{pMOS}}\right)$ (AVG $\left.\left.\left(\Delta \mathrm{V}_{\mathrm{TH}, \mathrm{pFET}}\right)\right)\right)$ to the nominal threshold voltage of the pMOS (pFET) (e.g., $\mathrm{V}_{\mathrm{TH}, \mathrm{pMOS}}$ $\left.\left(\mathrm{V}_{\mathrm{TH}, \mathrm{PFET}}\right)\right)$ under three different supply voltage levels and different time durations for the KSA and RCA structures. As the results indicate, the maximum (minimum) NBTI delay induced degradation belongs to the nominal (near-threshold) supply voltage level due to the strong dependence of the NBTI impact on the vertical electrical field of the transistor channel [64]. The figure shows that the impact of the NBTI on the transistors of the KSA structure is more than that of the RCA structure at all supply voltage levels. Note that the percentage difference between the pMOS (pFET) transistors threshold voltage drifts versus the time for these two structures is more or less unchanged for different supply voltage levels. 


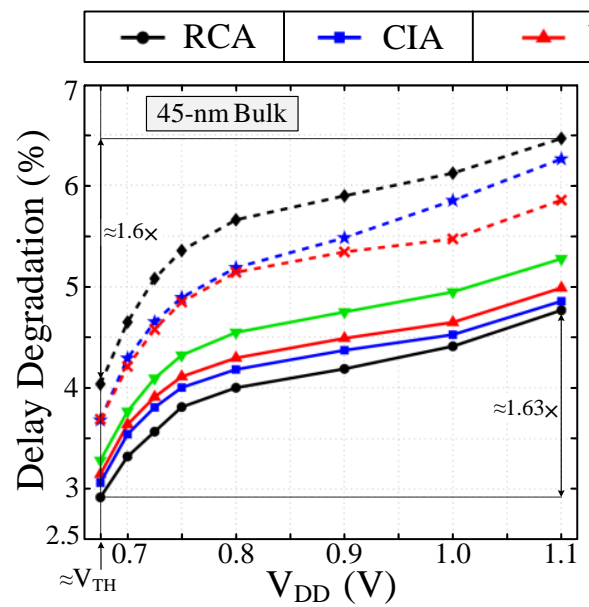

(a) After 3 years

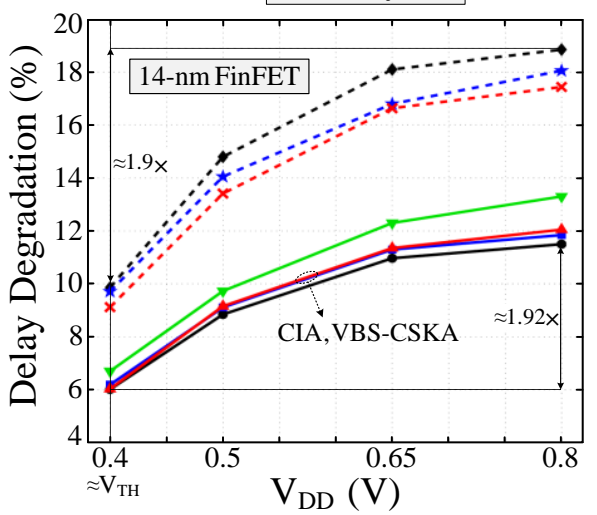

(d) After 3 years

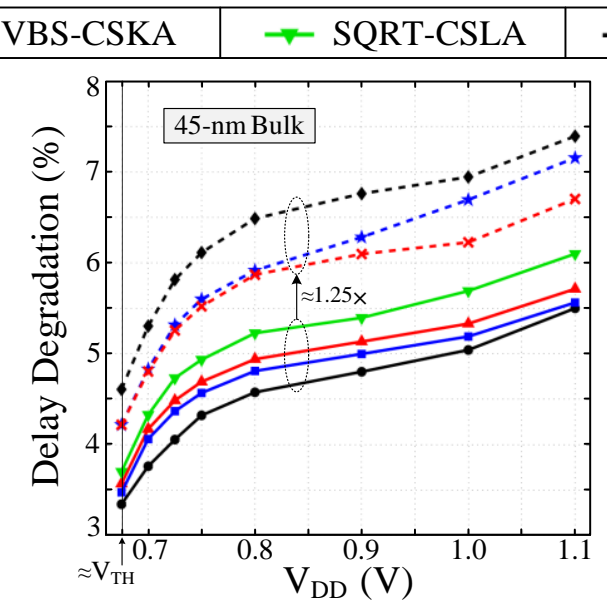

(b) After 5 years

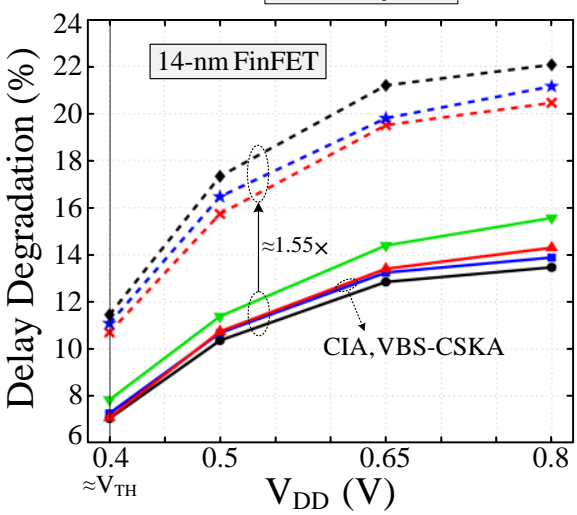

(e) After 5 years

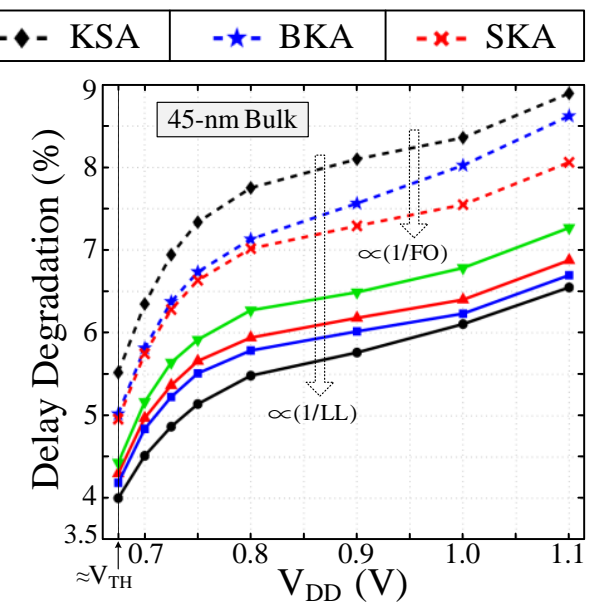

(c) After 10 years

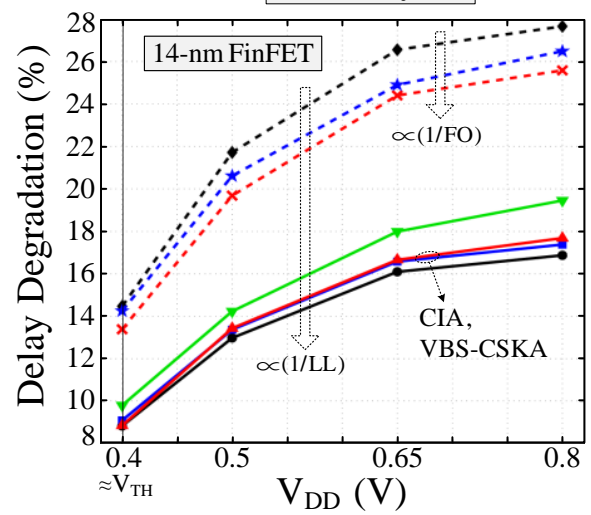

(f) After 10 years

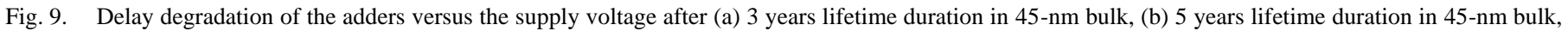

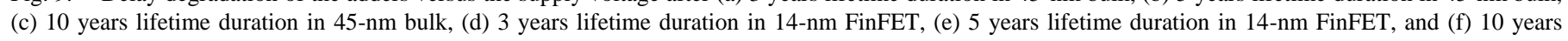
lifetime duration in 14-nm FinFET technologies at the room temperature of $25^{\circ} \mathrm{C}$.

The NBTI aging of an adder unit is a function of its structure (including internal cells, wiring tracks, and network nodes) which influences the propagation of a given data from the primary inputs to the primary outputs. For a given input data, the structure gives rise to different switching activities and stress levels inducing different threshold voltage shifts, and hence, timing path delays [35]-[37]. In Fig. 9, we have presented the results for the NBTI induced delay degradation of the adders versus the supply voltage after three predefined lifetime durations $(3,5$, and 10 years) in both technologies. The results reveal that the delay degradation rate decreases as the supply voltage is increased. It is due to the logarithmic dependence of the delay degradation to the gate overdrive (i.e., $\left(V_{D D}-V_{T H}\right)$ ). More specifically, reducing the supply voltage from the nominal voltage to the near-threshold voltage causes, on average on adder structures, about 1.6 (1.9) fold decrease in the delay degradation of the adders implemented in the 45-nm bulk (14-nm FinFET) technology. Also, the RCA (KSA) has the minimum (maximum) NBTI induced delay degradation for the whole supply voltage range and lifetime durations. Note that the PPAs have higher delay degradations compared to those of the CPAs. The average delay degradation of the PPAs is about $1.25 \mathrm{x}(1.55 \mathrm{x})$ larger than that of the CPAs in the 45-nm bulk (14-nm FinFET) technology for the whole supply voltage range and lifetime durations. Also, among the PPAs (CPAs), the KSA (SQRT-CSLA) has the highest delay degradation, while the SKA (RCA) has the smallest delay degradation. As mentioned before, the architectural parameters such as logic level (LL) and fan-out (FO) of the adder structure define the internal nodes and wiring tracks of the adders. These parameters have the main effect on the speed, power, and area usage of the adders as well as the delay degradation trend of the adder structures (due to the NBTI aging). The LL parameter has the main effect on the amount of delay degradation of the adder structures, which the KSA (RCA) with the smallest (largest) logic level, has the maximum (minimum) delay degradation compared to that of other adders. This means that the delay degradation of the adders is proportional to (1/LL) of their topologies as has been stated in [35]. Along with the LL, the FO parameter can be used also to justify the delay degradation of the adders. As the results shown in Fig. 9, the SKA with the highest FO (the SKA with the fan-out of 17 compared to the fan-out of 2 of the KSA and BKA) has the smallest delay degradation among the PPAs. The delay degradation of the adders is also proportional to $(1 / \mathrm{FO})$ of their topologies as has been stated in [35]. Although the LL of the BKA is larger than that of the SKA (i.e., 9>5), however, the FO of the SKA is considerably larger 


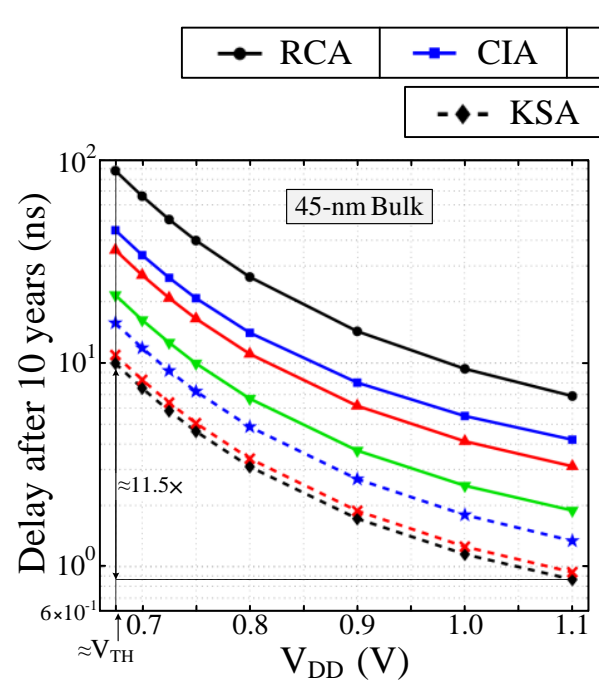

(a) \begin{tabular}{l|l|l|}
$\leftarrow$ VBS-CSKA & $\rightarrow$ SQRT-CSLA \\
\hline$-\star-$ BKA & $-x-$ SKA
\end{tabular}

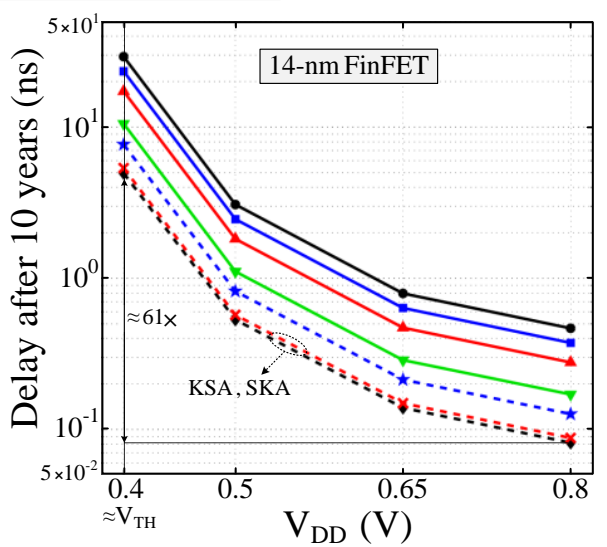

(b)

Fig. 10. Critical path delay of the adder structures after 10 years lifetime versus the supply voltage for (a) 45-nm bulk and (b) 14-nm FinFET technologies.

than that of the BKA (i.e., $17>2$ ). Hence, the delay degradation of the SKA is slightly smaller than that of the BKA. In the CPA structures, the difference between the FO parameters is very low (almost similar), while the difference between the LL parameters is considerable. Hence, the RCA (SQRT-CSLA) with the largest (smallest) LL has the lowest (highest) NBTI induced delay degradation. Thus, the NBTI induced delay degradations of the adder structures for the whole range of the supply voltage (from superthreshold voltage region to near-threshold voltage region) are observed to be a function of LL and FO of its topology regardless of the lifetime durations.

Finally, the critical path delays of the adders after 10 years have been drawn in Fig. 10 which shows that the RCA (KSA) remain the slowest (fastest) structure among the adders even in the presence of the NBTI aging effect. The comparison between the delays reported in Fig. 4 and Fig. 10 shows that the differences between the delays of the adders have slightly changed after 10 years. Also, in the $45-\mathrm{nm}$ bulk (14-nm FinFET) technology, the delays of adders after 10 years is, on average on adder structures, about 11.5 (61) times more if instead of the nominal voltage, the near-threshold voltage is used. This is similar to the results reported in Fig. 4. In the next section, a discussion on comparing the studied adders based on their performance and reliability parameters will be presented.

\section{ADDERS EFFICIENCY COMPARISON}

In this section, we take the approach of comparing different adders by defining a merit factor (MF) based on the performance and reliability parameters. The main parameters are delay $(D)$, power consumption $(P)$, normalized delay changes (variations) due to process variation $\left(\Delta D_{\text {norm,PV }}\right)$, and normalized NBTI aging induced delay degradation $\left(\Delta D_{\text {norm,NBTI }}\right)$. The delays and power consumptions of the adder structures at each supply voltage may be normalized by dividing them to the maximum value of the delay and power consumption of all the adders in each voltage level. We denote them by $D_{\text {norm }}$ and $P_{\text {norm }}$, respectively. Based on the results presented in Section IV, the delay (power consumption) of the RCA (KSA), at each supply voltage level, has the maximum value among the adder structures. Since, the speed (delay) and energy consumption are the key parameters for determining the efficiency of the adder, the product of the delay $\left(D_{\text {norm }}\right)$ and energy $\left(D_{\text {norm }} \times P_{\text {norm }}\right)$ are considered for determining the merit factor. Also, the amounts of $\Delta D_{\text {norm,PV }}\left(\Delta D_{\text {norm,NBTI }}\right)$ of the adders at each supply voltage are calculated by dividing the delay variations (i.e., $\sigma / \mu)$ due to process variation (NBTI aging induced delay degradations) to the maximum value of the delay variation (degradation) of all the adders in each voltage level. As investigated in Section V, the KSA, at each supply voltage level, has the maximum value of delay variation due to process variation and NBTI aging induced delay degradation among the adder structures. Based on the given explanations, we use the following MF relation:

$$
\mathrm{MF}=\frac{1}{D_{\text {norm }} \times\left(D_{\text {norm }} \times P_{\text {norm }}\right) \times \Delta D_{\text {norm }, \mathrm{PV}} \times \Delta D_{\text {norm }, \mathrm{NBTI}}} .
$$

Of course, depending on the importance weights of the parameters for an application, other merit functions may be defined. For the merit function defined above, a higher MF corresponds to a better adder structure.

The merit factor (MF) of the adder structures versus the supply voltage for 45-nm bulk and 14-nm FinFET technologies are shown in Fig. 11. Based on this figure, by decreasing the supply voltage, this factor (i.e., MF) decreases for all the adders in both bulk and FinFET technologies. However, for all the adders, at low supply voltages (around the near-threshold voltage), the factor start increasing after passing a minimum value. It should be noted that the ratios and trends of the performance and reliability parameters for the adder structures in the FinFET technology are different than those in the bulk technology for the supply voltage range considered in this work. Therefore, the MF values of the 


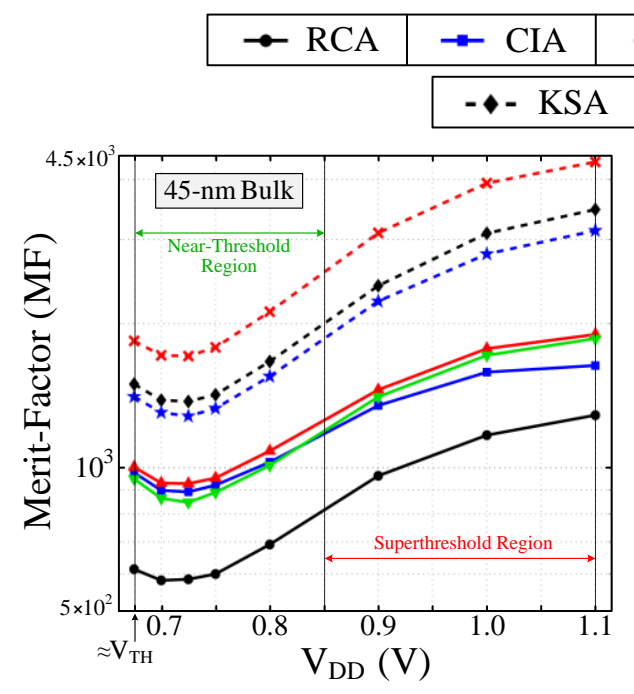

(a)

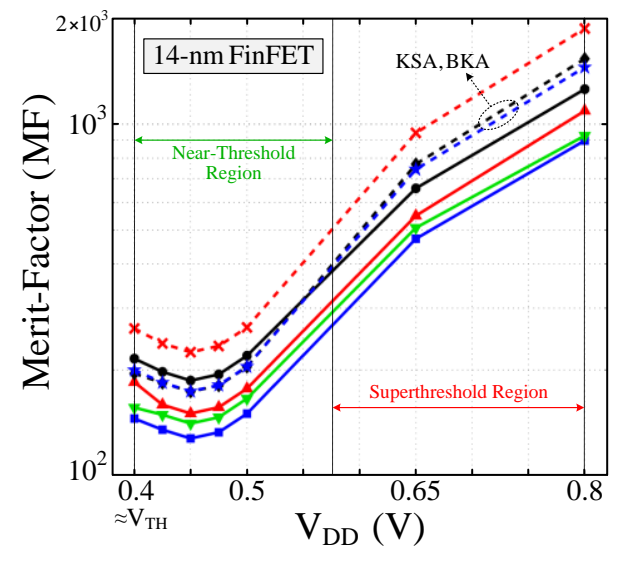

(b)

Fig. 11. Merit factor (MF) of the adders versus the supply voltage for (a) 45-nm bulk and (b) 14-nm FinFET technologies.

adders (and their trends versus the supply voltage) in the FinFET technology are also different.

The results indicate that reducing the voltage from $1.1 \mathrm{~V}$ $(0.8 \mathrm{~V})$ to the near-threshold voltage causes, on average, about 2.2 (7.1) fold decrease in the MF value for all adders in the 45nm bulk (14-nm FinFET) technology. For both technologies, the SKA structure has the highest MF among the adder structures. In the $45-\mathrm{nm}$ bulk, next to the SKA are KSA and BKA while RCA has the smallest MF. Also, for this technology, among the other CPAs, the VBS-CSKA (CIA) has the highest (smallest) MF in the super-threshold region, while in the near-threshold region, the SQRT-CSLA has the smallest MF among these adders. In the 14-nm FinFET technology, next to the SKA structure, are the KSA and BKA have the highest MF values in the superthreshold while the RCA has the highest value in the near-threshold region. Also, the CIA has the smallest MF value for the whole range of the supply voltage. These MF characteristics for the adder structures may be accounted for by considering the characteristics of the delay, power, and delay changes due to the NBTI-induced and process variations presented in Sections IV and V.

\section{CONCLUSION}

In this paper, a comparative study on the performance and reliability of 32-bit binary adder structures under a wide range of supply voltage levels was performed. For this study, several adder structures from carry propagate adders and parallel prefix adders were chosen. The supply voltage range included the operations of the adders in both the near- and superthreshold regions. The investigation, which was performed for both $45-\mathrm{nm}$ bulk and 14-nm FinFET technologies, included the performance parameters of delay, power, energy, and energy-delay-product parameters. Additionally, the impacts of the process variation on the time zero delay and negative bias temperature instability (NBTI) on the delay degradation after a given lifetime for a wide range of supply voltage were studied.
Finally, the betterness of the adders were compared using a merit factor (MF) based on their performance and reliability parameters for the whole range of the supply voltage. The results revealed that the reliability parameters similar to the performance parameters are a function of the adder architectures and those were the key components to determine the efficiencies of the adders. In addition, the results shown that the impacts of the process variation and NBTI on the delays of the high performance PPA structures were more than those of the CPA structures for the whole range of the supply voltage. The PPA structures, however, had the higher merit factors compared to the CPA structures under a wide range of supply voltage levels.

\section{ACKNOWLEDGMENT}

The work of M. Bahadori, M. Kamal, and A. Afzali-Kusha was supported by the Iranian National Science Foundation.

\section{REFERENCES}

[1] I. Koren, Computer Arithmetic Algorithms, 2nd edition A. K. Peters, Ltd., Natick, MA, 2002.

[2] R. Zimmermann, "Binary adder architectures for cell-based VLSI and their synthesis," Ph.D. dissertation, Dept. Inf. Technol. Elect. Eng., Swiss Federal Inst. Technol. (ETH), Zürich, Switzerland, 1998.

[3] S. Ghosh, D. Mohapatra, G. Karakonstantis, and K. Roy, "Voltage scalable high-speed robust hybrid arithmetic units using adaptive clocking," IEEE Transactions on Very Large Scale Integration (VLSI) Systems, vol. 18, no. 9, pp. 1301-1309, September 2010.

[4] R. G. Dreslinski, M. Wieckowski, D. Blaauw, D. Sylvester, and T. Mudge, "Near-threshold computing: Reclaiming Moore's law through energy efficient integrated circuits," Proceedings of the IEEE, vol. 98, no. 2, pp. 253-266, February 2010.

[5] S. Ghosh and K. Roy, "Parameter variation tolerance and error resiliency: new design paradigm for the nanoscale era," Proceedings of the IEEE, vol. 98, no. 10, pp. 1718-1751, October 2010.

[6] S. Seo et al., "Process variation in near-threshold wide SIMD architectures," 49th ACM/EDAC/IEEE Design Automation Conference (DAC), pp. 980-987, 3-7 June 2012. 
[7] D. Markovic, C. C. Wang, L. P. Alarcon, L. Tsung-Te, J. M. Rabaey, "Ultralow-Power Design in Near-Threshold Region," Proceedings of the IEEE, vol. 98, no. 2, pp. 237-252, February 2010.

[8] S. Jain et al., "A $280 \mathrm{mV}$-to-1.2V wide-operating-range IA-32 processor in 32nm CMOS," IEEE International Solid-State Circuits Conference Digest of Technical Papers (ISSCC 2012), pp. 66-68, 19-23 February 2012.

[9] S. K. Mathew, M. Anders, B. Bloechel, T. Nguyen, R. Krishnamurthy, and S. Borkar, "A 4-GHz 300-mW 64-bit integer execution ALU with dual supply voltages in 90-nm CMOS," IEEE Journal of Solid-State Circuits, vol. 40, no. 1, pp. 44-51, January 2005.

[10] B. Ramkumar, and H. M. Kittur, "Low-power and area-efficient carry select adder," IEEE Transactions on Very Large Scale Integration (VLSI) Systems, vol. 20, no. 2, pp. 371-375, February 2012.

[11] C. Nagendra, M. J. Irwin, and R. M. Owens, "Area-time-power tradeoffs in parallel adders," IEEE Transactions on Circuits and Systems II: Analog and Digital Signal Processing, vol. 53, no. 10, pp. 689-702, October 1996.

[12] Y. He and C. H. Chang, "A Power-Delay Efficient Hybrid CarryLookahead/Carry-Select Based Redundant Binary to Two's Complement Converter," IEEE Transactions on Circuits and Systems I: Regular Papers, vol. 55, no. 1, pp. 336-346, February 2008.

[13] C. H. Chang, J. Gu, and M. Zhang, "A review of 0.18um full adder performances for tree structured arithmetic circuits," IEEE Transactions on Very Large Scale Integration (VLSI) Systems, vol. 13, no. 6, pp. 686-695, June 2005.

[14] D. Patil, O. Azizi, M. Horowitz, R. Ho, and R. Ananthraman, "Robust energy-efficient adder topologies," 18th IEEE Symposium on Computer Arithmetic (ARITH'07), pp.16-28, 25-27 June 2007.

[15] R. UMA, V. Vijayan, M. Mohanapriya, and S. Paul, "Area, delay and power comparison of adder topologies," International Journal of VLSI design \& Communication Systems, vol. 3, no. 1, February 2012.

[16] A. N. Jayanthi and C. S. Ravichandran, "Comparison of performance of high speed VLSI adders," International Conference on Current Trends in Engineering and Technology, pp. 99-104, 3-3 July 2013.

[17] V. G. Oklobdzija, B. R. Zeydel, H. Dao, S. Mathew, and R. Krishnamurthy, "Energy-delay estimation technique for highperformance microprocessor VLSI adders," Proceedings of the 16th IEEE Symposium on Computer Arithmetic, pp. 272-279, June 2003.

[18] V. G. Oklobdzija, B. R. Zeydel, H. Dao, S. Mathew, and R. Krishnamurthy, "Comparison of high-performance VLSI adders in energy-delay space," IEEE Transactions on Very Large Scale Integration (VLSI) Systems, vol. 13, no. 6, pp. 754-758, June 2005.

[19] M. Vratonjic, B. R. Zeydel, and V. G. Oklobdzija, "Low- and ultra low-power arithmetic units: Design and comparison," in Proceeding of IEEE International Conference on Computer Design (ICCD): VLSI in Computers and Processors, pp. 249-252, 2-5 October 2005.

[20] R. Zlatanovici, S. Kao, and B. Nikolic, "Energy-delay optimization of 64-bit carry-lookahead adders with a 240 ps $90 \mathrm{~nm}$ CMOS design example," IEEE Journal of Solid-State Circuits (JSSC), vol. 44, no. 2, pp. 569-583, February 2009.

[21] B. R. Zeydel, D. Baran, and V. G. Oklobdzija, "Energy-efficient design methodologies: High-performance VLSI adders," IEEE Journal of Solid-State Circuits (JSSC), vol. 45, no. 6, pp. 1220-1233, June 2010.

[22] J. F. Lin, Y. T. Hwang, M. H. Sheu, and C. C. Ho, "A novel highspeed and energy efficient 10-transistor full adder design," IEEE Transactions on Circuits and Systems I: Regular Papers, vol. 54, no. 5, pp. 1050-1059, May 2007.

[23] I. Hassoune, D. Flandre, I. O'Connor, and J. Legat, "ULPFA: a new efficient design of a power-aware full adder," IEEE Trans. on Circuits Systems I: Regular Papers, vol. 57, no. 8, pp. 2066-2074, Aug. 2010.

[24] W. Ibrahim and V. Beiu, "Threshold voltage variations make full adders reliabilities similar," IEEE Transactions on Nanotechnology, vol. 9, no. 6, pp. 664-667, November 2010.

[25] W. Ibrahim, A. Beg, and V. Beiu, "Highly reliable and low-power full adder cell," 11th IEEE Conference on Nanotechnology (IEEE-NANO), pp. 500-503, 15-18 August 2011.

[26] J. Hu and X. Yu, "Near-threshold full adders for ultra low-power applications," Second IEEE Pacific-Asia Conference on Circuits, Communications and System (PACCS), pp. 300-303, 1-2 August 2010.

[27] A. Islam, M. W. Akram, A. Imran, and M. Hasan, "Energy efficient and process tolerant full adder design in near threshold region using FinFET," IEEE International Symposium on Electronic System Design (ISED), pp. 56-60, 20-22 December 2010.
[28] D. Mohapatra, G. Karakonstantis, K. Roy, "Low-power processvariation tolerant arithmetic units using input-based elastic clocking," ACM/IEEE International Symposium on Low Power Electronics and Design (ISLPED), pp. 74-79, 27-29 August 2007.

[29] Y. Chen, H. Li, J. Li, and C. Koh, "Variable-latency adder (VL-adder): New arithmetic circuit design practice to overcome NBTI," ACM/IEEE International Symposium on Low Power Electronics and Design (ISLPED), pp. 195-200, 27-29 August 2007.

[30] Y. Chen, H. Li, C. Koh, G. Sun, J. Li, Y. Xie, and K. Roy, "Variablelatency adder (VL-adder) designs for low power and NBTI tolerance," IEEE Transactions on Very Large Scale Integration (VLSI) Systems, vol. 18, no. 11, pp. 1621-1624, November 2010.

[31] K. Du, P. Varman, and K. Mohanram, "High performance reliable variable latency carry select addition," Design, Automation and Test in Europe Conference and Exhibition (DATE), pp. 1257-1262, 12-16 March 2012.

[32] M. Bahadori, M. Kamal, A. Afzali-Kusha, and M. Pedram, "Highspeed and energy-efficient carry skip adder operating under a wide range of supply voltage levels," To appear in IEEE Transactions on Very Large Scale Integration (VLSI) Systems, 2015.

[33] Y. H. Cho, I. C. Lin, and Y. M. Yang, "Aging-aware reliable multiplier design," IEEE International SOC Conference (SOCC), pp. 322-327, 12-14 September 2012.

[34] I. C. Lin, Y. H. Cho, and Y. M. Yang, "Aging-aware reliable multiplier design with adaptive hold logic," IEEE Transactions on Very Large Scale Integration (VLSI) Systems, vol. 23, no. 3, pp. 544-556, March 2015.

[35] H. Kukner et al., "NBTI aging on 32-Bit adders in the downscaling planar FET technology nodes," 17th IEEE Euromicro Conference on Digital System Design (DSD), pp. 98-107, 27-29 August 2014.

[36] H. Kukner et al., "Degradation analysis of datapath logic subblocks under NBTI aging in FinFET technology," 15th IEEE International Symposium on Quality Electronic Design, pp. 473-479, 3-5 Mar. 2014.

[37] T. An, H. Cai, and L. A. de Barros Naviner, "Simulation study of aging in CMOS binary adders," 37th IEEE International Convention on Information and Communication Technology, Electronics and Microelectronics (MIPRO), pp. 51-55, 26-30 May 2014.

[38] P. M. Kogge and H. Stone, "A parallel algorithm for the efficient solution of a general class of recurrence equations," IEEE Transactions on Computers, vol. C-22, no. 8, pp. 786-793, August 1973.

[39] R. P. Brent and H. Kung, "A regular layout for parallel adders," IEEE Transactions on Computers, vol. C-31, no. 3, pp. 260-264, Mar. 1982.

[40] J. Sklansky, "Conditional-sum addition logic," IRE Transactions on Electronic Computers, vol. EC-9, no. 2, pp. 226-231, June 1960.

[41] D. Harris, "A taxonomy of parallel prefix networks," IEEE Conference Record of the Thirty-Seventh Asilomar Conference on Signals, Systems and Computers, vol. 2, pp. 2213-2217, November 2003.

[42] A. Guyot, B. Hochet, and J. Muller, "A way to build efficient carryskip adders," IEEE Transactions on Computers, vol. C-36, no. 10, pp. 1144-1152, October 1987.

[43] M. Alioto and G. Palumbo, "A simple strategy for optimized design of one-level carry-skip adders," IEEE Transactions on Circuits and Systems I: Fundamental Theory and Applications, vol. 50, no. 1, pp. 141-148, January 2003.

[44] M. Alioto, G. Palumbo, and M. Poli, "Optimized design of parallel carry-select adders," Integration, the VLSI Journal, Elsevier, vol. 44, Issue 1, pp. 62-74, January 2011.

[45] 14 nm LSTP FinFET Predictive Technology Model (PTM). [Online]. Available: http://www.ptm.asu.edu, accessed June 2012.

[46] NanGate $45 \mathrm{~nm}$ Open Cell Library. [Online]. Available: http//www.nangate.com, accessed Dec. 2010.

[47] Cadence SOC Encounter. [Online]. Available: http//www.cadence.com, version 10.1.

[48] Synopsys HSPICE. [Online]. Available: http//www.synopsys.com, accessed Dec. 2013.

[49] J.-P. Colinge, FinFETs and Other Multi-Gate Transistors, Springer, 2007.

[50] D. Hisamoto et al., "FinFET-a self-aligned double-gate MOSFET scalable to $20 \mathrm{~nm}$," IEEE Transactions on Electron Devices, vol. 47, no. 12 pp. 2320-2325, December 2000.

[51] G. Leung, L. Lai, P. Gupta, and C. O. Chui, "Device- and circuit-level variability caused by line edge roughness for sub-32-nm FinFET technologies," IEEE Transactions on Electron Devices, vol. 59, no. 8, pp. 2057-2063, August 2012. 
[52] E. Baravelli et al., "Impact of LER and random dopant fluctuations on FinFET matching performance," IEEE Transactions on Nanotechnology, vol. 7, no. 3, pp. 291-298, May 2008.

[53] Y. Ye et al., "Random variability modeling and its impact on scaled CMOS circuits," Journal of Computational Electronics, vol. 9, no. 3-4, pp. 108-113, October 2010.

[54] H. Nam et al., "Analysis of random variations and variation-robust advanced device structures", Journal of Semiconductor Technology and Science, vol. 14, no. 1, pp. 8-22, 2014.

[55] K. Bernstein et al., "High-performance CMOS variability in the 65-nm regime and beyond," IBM Journal of Research and Development, vol. 50, no. 4.5, pp. 433-449, July 2006.

[56] K. Kuhn et al., "Managing process variation in Intel's 45nm CMOS technology," Intel Technology Journal, vol. 12, Issue 2, pp. 93-109, May 2008.

[57] K. J. Kuhn, "Reducing variation in advanced logic technologies: approaches to process and design for manufacturability of nanoscale CMOS," IEEE International Electron Devices Meeting (IEDM), pp. 471-474, 10-12 December 2007.

[58] S. Banerjee, P. Elakkumanan, D. Chidambarrao, J. Culp, and M. Orshansky, "Analysis of systematic variation and impact on circuit performance," SPIE Advanced Lithography-International Society for Optics and Photonics, pp. 69250K-69250K, March 2008.

[59] M. Orshansky, S. Nassif, and D. Boning, Design for Manufacturability and Statistical Design, Springer, 2008.

[60] D. Sylvester, K. Agarwal, and S. Shah, "Variability in nanometer CMOS: Impact, analysis, and minimization," Integration, the VLSI Journal, Elsevier, vol. 41, Issue 3, pp. 319-339, May 2008.

[61] M. Kamal, A. Afzali-Kusha, S. Safari, and M. Pedram, "Design of NBTI resilient extensible processors," Integration, the VLSI Journal, Elsevier, vol. 49, pp. 22-34, March 2015.

[62] B. Tudor et al., "MOSRA: An efficient and versatile MOS aging modeling and reliability analysis solution for $45 \mathrm{~nm}$ and below," 10th IEEE International Conference on Solid-State and Integrated Circuit Technology (ICSICT), pp. 1645-1647, 1-4 November 2010.

[63] [Online]. Available: https://www.synopsys.com/Tools/Verification/ AMSVerification/CircuitSimulation/HSPICE/Documents/mosra-wp.pdf

[64] B. C. Paul, K. Kang, H. Kufluoglu, M. A. Alam, and K. Roy, "Impact of NBTI on the temporal performance degradation of digital circuits," IEEE Electron Device Letters, vol. 26, no. 8, pp. 560-562, Aug. 2005.

[65] Synopsys Design Compiler. [Online]. Available: www.synopsys.com.

[66] NanGate $15 \mathrm{~nm}$ FinFET Open Cell Library. [Online]. Available: http//www.nangate.com, accessed May 2014.

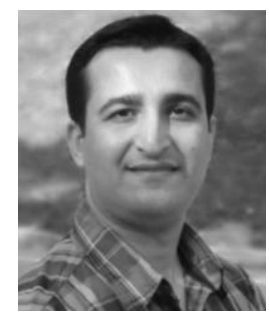

Milad Bahadori received the M.Sc. degree from the Sharif University of Technology, Tehran, Iran, in 2011, and the Ph.D. degree from the University of Tehran, Tehran, Iran, in 2015, all in in electrical and electronic engineering.

He was a Research Assistant with the Sharif Integrated Circuits Design Center, Sharif University of Technology, from 2009 to 2012, where he was involved in digital systems design. $\mathrm{He}$ joined the Low-Power High-Performance Nanosystems Laboratory, University of Tehran, in 2012, as a Research Assistant. His current research interests include low-power high-performance VLSI design, reliability in nanoscale design, near-threshold computing, and high-performance low power arithmetic circuits design.

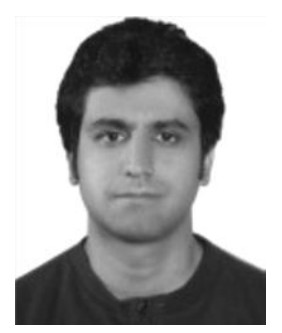

Mehdi Kamal received the B.Sc. degree from the Iran University of Science and Technology, Tehran, Iran, in 2005, the M.Sc. degree from the Sharif University of Technology, Tehran, in 2007, and the Ph.D. degree from the University of Tehran, Tehran, in 2013, all in computer engineering.

$\mathrm{He}$ is currently the assistant professor with the School of Electrical and Computer Engineering of the University of Tehran, Iran. His current research interest include reliability in nanoscale design, application specific instruction set processor design, hardware/software codesign, and low-power design.

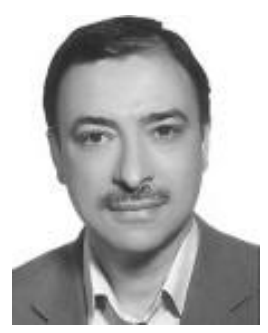

Ali Afzali-Kusha received the B.Sc. degree from the Sharif University of Technology, Tehran, Iran, in 1988, the M.Sc. degree from the University of Pittsburgh, Pittsburgh, PA, USA, in 1991, and the Ph.D. degree from the University of Michigan, Ann Arbor, MI, USA, in 1994, all in electrical engineering.

He was a Post-Doctoral Fellow with the University of Michigan from 1994 to 1995 . He has been with the University of Tehran, since 1995, where he is currently a Professor of the School of Electrical and Computer Engineering and the Director of the Low-Power High-Performance Nanosystems Laboratory. He was a Research Fellow with the University of Toronto, Toronto, ON, Canada, and the University of Waterloo, Waterloo, ON, Canada, in 1998 and 1999, respectively. His current research interests include low-power high-performance design methodologies from the physical design level to the system level for nanoelectronics era.

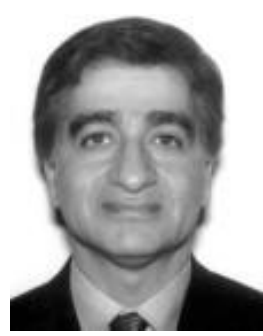

Massoud Pedram received the Ph.D. degree in electrical engineering and computer sciences from the University of California at Berkeley, Berkeley, CA, USA, in 1991. He is currently the Stephen and Etta Varra Professor with the Ming Hsieh Department of Electrical Engineering, University of Southern California, Los Angeles, CA, USA. He holds 10 U.S. patents and has authored four books, 12 book chapters, and over 140 archival and 350 conference papers.

His current research interests include low-power electronics, energyefficient processing, and cloud computing to photovoltaic cell power generation, energy storage, and power conversion, and RT level optimization of VLSI circuits to synthesis and physical design of quantum circuits.

Prof. Pedram and his students have received six conference and two IEEE Transactions Best Paper Awards for the research. He was a recipient of the 1996 Presidential Early Career Award for Scientists and Engineers and an ACM Distinguished Scientist, and currently serves as the Editor-in- Chief of the ACM Transactions on Design Automation of Electronic Systems. He has served on the Technical Program Committee of a number of premiere conferences in his field. He was the Founding Technical Program Co Chair of the 1996 International Symposium on Low-Power Electronics and Design and the Technical Program Chair of the 2002 International Symposium on Physical Design. 